\title{
Unification of SUSY breaking and GUT breaking
}

\author{
Tatsuo Kobayashi ${ }^{a}$ and Yuji Omura ${ }^{b}$ \\ ${ }^{a}$ Department of Physics, Hokkaido University, \\ Sapporo 060-0810, Japan \\ ${ }^{b}$ Department of Physics, Nagoya University, \\ Nagoya 464-8602, Japan \\ E-mail: kobayashi@particle.sci.hokudai.ac.jp, \\ yujiomur@eken.phys.nagoya-u.ac.jp
}

ABSTRACT: We build explicit supersymmetric unification models where grand unified gauge symmetry breaking and supersymmetry (SUSY) breaking are caused by the same sector. Besides, the SM-charged particles are also predicted by the symmetry breaking sector, and they give the soft SUSY breaking terms through the so-called gauge mediation. We investigate the mass spectrums in an explicit model with $\mathrm{SU}(5)$ and additional gauge groups, and discuss its phenomenological aspects. Especially, nonzero A-term and B-term are generated at one-loop level according to the mediation via the vector superfields, so that the electro-weak symmetry breaking and $125 \mathrm{GeV}$ Higgs mass may be achieved by the large B-term and A-term even if the stop mass is around $1 \mathrm{TeV}$.

Keywords: Supersymmetry Breaking, Beyond Standard Model, Extended Supersymmetry, Gauge Symmetry

ARXIV EPRINT: 1410.5173 


\section{Contents}

1 Introduction 1

$2 \mathrm{SU}(N) \times \mathrm{SU}\left(N_{F}\right)_{F} \times \mathrm{U}(1)_{\phi}$ gauge theory 3

2.1 Gauge bosons 4

2.2 SM-charged fields from symmetry breaking sector 5

2.3 RG flows of the gauge couplings 5

$\begin{array}{ll}2.4 \text { Soft SUSY breaking terms } & 7\end{array}$

$3 \mathrm{SU}(5)_{F} \times \mathrm{SU}(2) \times \mathrm{U}(1)_{\phi}$ gauge theory: $\left(N_{F}, N\right)=(5,2) \quad 9$

3.1 Gauge couplings 10

$\begin{array}{lll}3.2 & \text { Soft SUSY breaking terms } & 11\end{array}$

$\begin{array}{lll}3.3 & \text { Shift of the gluino mass } & 13\end{array}$

3.4 Consistency with the Higgs mass and the EW symmetry breaking 14

$4 \mathrm{SU}(5)_{F} \times \mathrm{SU}(3) \times \mathrm{U}(1)_{\phi}$ gauge theory: $\left(N_{F}, N\right)=(5,3) \quad 15$

5 Summary 16

A Mass spectrums of the particles in the symmetry breaking sector $\quad 17$

$\begin{array}{ll}\text { B Concrete parameter set } & 19\end{array}$

\section{Introduction}

As well-known, the Standard Model (SM) is very successful in describing our nature, and it is firmly established by the Higgs discovery at the LHC $[1,2]$. There are still some ambiguities in not only the signal strength of the Higgs particle but also the other observations such as flavor physics, but it would be getting more difficult to consider new-physics effects in any signals.

On the other hand, we are sure that the SM remains several mysteries about our nature: the origin of the fermion generations, the hyper-charge assignment, the Higgs mass, and so on. Many Beyond Standard Models (BSM) were proposed so far motivated by those mysteries, and some of them are expected to be found near future. One of the candidates is the supersymmetric grand unified theory (GUT), which reveals the origin of the Higgs mass and the fermion charges. There are some issues in Yukawa couplings, for instance, how to generate realistic Yukawa couplings and heavy colored Higgs, but it succeeds in the charge quantization $\left(\left|Q_{e}+Q_{p}\right|<10^{-21}[3]\right)$ and naturally deriving the electro-weak (EW) scale, if the supersymmetry (SUSY) scale $\left(\Lambda_{\mathrm{SUSY}}\right)$ is close to the EW scale. The supersymmetric GUT scenario is constrained by the observation of the proton 
decay, the direct search of SUSY particles, and the SM measurements. Especially, the Higgs discovery around $125 \mathrm{GeV}$ may require high-scale SUSY $\left(\Lambda_{\mathrm{SUSY}} \gg O(1) \mathrm{TeV}\right)[4,5]$, which may discard the strong motivation of SUSY, that is, the natural explanation of the EW scale. Furthermore, the gauge coupling unification of supersymmetric SU(5) GUT might be lost in high-scale SUSY, depending on the mass spectrum of the SUSY particles. The supersymmetric models could have so many parameters in the bottom-up approach, so that we could have some solutions for the Higgs mass and the gauge coupling unification. However, it is very important to find how to derive such a specific SUSY mass spectrum.

In this paper, we propose an explicit supersymmetric GUT with $\mathrm{SU}(5)_{F} \times \mathrm{SU}(2) \times \mathrm{U}(1)_{\phi}$ gauge groups. We discard the miracle of the gauge coupling unification in the Minimal Supersymmetric SM (MSSM), but SUSY breaking and GUT breaking sectors are unified. ${ }^{1}$ The SM-charged particles also appear after the symmetry breaking, so the messenger fields for the gauge mediation is also introduced by the breaking sector in our model. ${ }^{2}$ The SM fields are only charged under the $\mathrm{SU}(5)_{F}$ gauge group, so that the charge quantization is realized.

The breaking sector consists of one $\mathrm{SU}(5)_{F}$ adjoint plus singlet filed $(\Phi)$ and $\mathrm{SU}(5)_{F}$ fundamental and anti-fundamental fields $(\phi, \widetilde{\phi})$. The vector-like pairs $(\phi, \widetilde{\phi})$ are also charged under $\mathrm{SU}(2) \times \mathrm{U}(1)_{\phi}$. As discussed in ref. [23], this type of gauge theory causes SUSY breaking along with the gauge symmetry breaking. In our model, $\mathrm{SU}(5)_{F} \times \mathrm{SU}(2) \times$ $\mathrm{U}(1)_{\phi}$ symmetry breaks down to the $\mathrm{SM}$ gauge groups, $\mathrm{SU}(3)_{c} \times \mathrm{SU}(2)_{L} \times \mathrm{U}(1)_{Y}$, where $\mathrm{SU}(3)_{c}$ is from the subgroup of $\mathrm{SU}(5)_{F}$, and $\mathrm{SU}(2)_{L} \times \mathrm{U}(1)_{Y}$ are the linear combinations of the subgroup of $\mathrm{SU}(5)_{F}$ and $\mathrm{SU}(2) \times \mathrm{U}(1)_{\phi}$. SUSY is broken by the F-component of the part of $\Phi$. After the symmetry breaking, SM-charged particles are generated by the fluctuation of $\Phi$ and $(\phi, \widetilde{\phi})$ around the vacuum expectation values (VEVs). One interesting point is that the massive gauge boson of $\mathrm{SU}(5)_{F}$ and the fermionic partners could mediate the SUSY breaking effect through the gauge coupling with $\Phi$, and play a crucial role in generating the non-zero A-term and B-term as discussed in refs. [24, 25]. It is well-known that SUSY-scale A-term could shift the upper bound on the lightest Higgs mass in the MSSM, even if squark is light, and the SUSY-scale B-term is required to realize the EW symmetry breaking. Our A-term and B-term are given at one-loop level, so that they are the same order as the squark masses and gaugino masses. In fact, we will see that Higgs mass could be around $125 \mathrm{GeV}$, even if $\Lambda_{\mathrm{SUSY}}$ is less than $O(1) \mathrm{TeV}$, and the B-term could be consistent with the EW symmetry breaking.

In section 2, we introduce the SUSY and GUT breaking sector in generic $\mathrm{SU}\left(N_{F}\right)_{F} \times$ $\mathrm{SU}(N) \times \mathrm{U}(1)_{\phi}$ gauge theory. There, we discuss not only the symmetry breaking, but also the behavior of the gauge couplings and soft SUSY breaking terms according to the gauge mediation with the mediators of the chiral superfields and the vector superfields. In section 3, we apply the breaking sector to the $\mathrm{SU}(5)_{F} \times \mathrm{SU}(2) \times \mathrm{U}(1)_{\phi}$ gauge theory. As we mentioned above, an interesting aspect of this model is the improvement of the consistency with the EW symmetry breaking and Higgs mass in the case with low-scale

\footnotetext{
${ }^{1}$ This type of scenario has been proposed in refs. [6-11].

${ }^{2}$ The messenger sector and SUSY breaking sector are unified, for instance, in refs. [12-22].
} 


\begin{tabular}{|c|ccc|}
\hline & $\phi$ & $\widetilde{\phi}$ & $\Phi$ \\
\hline $\mathrm{SU}\left(N_{F}\right)_{F}$ & $\mathbf{N}_{\mathbf{F}}$ & $\overline{\mathbf{N}_{\mathbf{F}}}$ & $\operatorname{adj}_{N_{F}}+\mathbf{1}$ \\
$\mathrm{SU}(N)$ & $\overline{\mathbf{N}}$ & $\mathbf{N}$ & $\mathbf{1}$ \\
$\mathrm{U}(1)_{\phi}$ & $Q_{\phi}$ & $-Q_{\phi}$ & 0 \\
\hline
\end{tabular}

Table 1. Chiral superfields in $\mathrm{SU}\left(N_{F}\right)_{F} \times \mathrm{SU}(N) \times \mathrm{U}(1)_{\phi}$ gauge theory.

SUSY. We investigate the soft SUSY breaking terms, and discuss how well it is achieved in our scenario. In section 4 , we give a comment on the possibility that the breaking sector is applied to other GUT models. Section 5 is devoted to the summary. In appendix A, we give the mass spectrum in the SUSY breaking sector. In appendix B, we show examples of mass spectrums in the MSSM sector.

\section{$2 \mathrm{SU}(N) \times \mathrm{SU}\left(N_{F}\right)_{F} \times \mathrm{U}(1)_{\phi}$ gauge theory}

In this section, we introduce the model which causes SUSY breaking together with gauge symmetry breaking, based on ref. [23].

We consider $\mathrm{SU}\left(N_{F}\right)_{F} \times \mathrm{SU}(N) \times \mathrm{U}(1)_{\phi}$ gauge theory with $N_{F}>N$. The matter content is shown in table 1: $\Phi$ is the $\mathrm{SU}\left(N_{F}\right)_{F}$ adjoint plus singlet field and $(\phi, \widetilde{\phi})$ pair is the vector-like under $\mathrm{SU}\left(N_{F}\right)_{F} \times \mathrm{SU}(N) \times \mathrm{U}(1)_{\phi}$ gauge group.

The superpotential is given by

$$
W_{R}=-h \operatorname{Tr}_{N}(\widetilde{\phi} \Phi \phi)+h \Lambda_{G} \operatorname{Tr}_{N_{F}}(\Phi),
$$

assigning $\mathrm{U}(1)_{R}$ symmetry: the R-charge of $\Phi$ is 2 and the R-charge of $(\phi, \widetilde{\phi})$ is vanishing. However, there would be an issue about how to break R-symmetry and how to avoid the massless particle according the $\mathrm{U}(1)_{R}$ symmetry breaking. Let us introduce explicit $\mathrm{U}(1)_{R}$ breaking terms,

$$
W_{\not R}=m_{\phi} \operatorname{Tr}_{N}(\tilde{\phi} \phi)+c,
$$

and discuss the superpotential as $W_{\mathrm{SB}}=W_{R}+W_{\not R}$. In ref. [23], $W_{R}$ is generated, considering the dual side of $\mathrm{SU}\left(N_{F}\right)_{F} \times \mathrm{SU}\left(N+N_{F}\right)$ gauge theory with the $N_{F}$ vector-like pairs $\left(q_{d}, \widetilde{q}_{d}\right)$ of $\mathrm{SU}\left(N+N_{F}\right)$ gauge group. $\Phi$ is interpreted as the composite operator as $\Phi \equiv \widetilde{q}_{d} q_{d}$, and $h \Lambda_{G} \operatorname{Tr}_{N_{F}}(\Phi)$ in $W_{R}$ corresponds to the mass term of the $\left(q_{d}, \widetilde{q}_{d}\right)$.

Some ideas to induce $W_{\not R}$ have been proposed in ref. [26], where the small wave-function factor of $\Phi$ suppresses $\Phi^{2}$ and $\Phi^{3}$ terms according to the strong dynamics or the profile in the extra dimension. In ref. [27], the effect of the explicit R-symmetry breaking terms is well studied. Here, we simply start the discussion from the superpotential $W_{\mathrm{SB}}$ assuming that such a mechanism, as discussed in ref. [26], works in underlying theories above the GUT, and study the symmetry breaking. In the global SUSY with canonical Kähler potential, the scalar potential is given by $V=\left|\partial_{\Phi} W_{\mathrm{SB}}\right|^{2}+\left|\partial_{\phi} W_{\mathrm{SB}}\right|^{2}+\left|\partial_{\widetilde{\phi}} W_{\mathrm{SB}}\right|^{2}$, and SUSY vacua satisfy $\partial_{\Phi} W_{\mathrm{SB}}=\partial_{\phi} W_{\mathrm{SB}}=\partial_{\widetilde{\phi}} W_{\mathrm{SB}}=0$. In this model, $\partial_{\Phi} W_{\mathrm{SB}}$ is given by

$$
\partial_{\Phi_{j i}} W_{\mathrm{SB}}=-h(\phi \widetilde{\phi})_{i j}+h \Lambda_{G} \delta_{i j}
$$


and all elements cannot be vanishing, because $N_{F} \times N_{F}$ matrix $(\phi \widetilde{\phi})$ has the rank $N$ $\left(<N_{F}\right)$. This means that SUSY is broken by the F-components of $\left(N_{F}-N\right)$ elements in $\Phi$ and $\mathrm{SU}\left(N_{F}\right)_{F}$ would be also broken.

Following ref. [23], we decompose $\Phi$ and $(\phi, \tilde{\phi})$ as

$$
\begin{aligned}
\Phi & =\left(\begin{array}{cc}
\left(v_{Y}\right) \mathbf{1}_{N}+\hat{Y} & \widetilde{Z} \\
Z & \left(v_{X}\right) \\
\mathbf{1}_{\widetilde{N}}+\hat{X}
\end{array}\right), \\
\phi & =\left(\begin{array}{c}
\left(v_{\chi}\right) \mathbf{1}_{N}+\hat{\chi} \\
\rho
\end{array}\right), \quad \widetilde{\phi}^{T}=\left(\begin{array}{c}
\left(v_{\chi}\right) \mathbf{1}_{N}+\hat{\tilde{\chi}}^{T} \\
\widetilde{\rho}^{T}
\end{array}\right),
\end{aligned}
$$

where $\hat{Y}, \hat{\chi}$ and $\hat{\tilde{\chi}}$ are $N \times N$ matrices, $\hat{X}$ is an $\tilde{N} \times \tilde{N}$ matrix $\left(\tilde{N}=N_{F}-N\right), Z$ and $\rho$ $(\widetilde{Z}$ and $\widetilde{\rho})$ are $N \times \widetilde{N}$ matrices $(\widetilde{N} \times N$ matrices $)$. The VEVs, $v_{Y}$ and $v_{\chi}$, are fixed by the stationary conditions

$$
\begin{aligned}
v_{Y} & =\frac{m_{\phi}}{h}, \\
v_{\chi} & =\Lambda_{G} .
\end{aligned}
$$

This solution also satisfies the D-flat conditions. $v_{X}$ is a flat direction in global SUSY. If we consider gravity and one-loop corrections, it would be stabilized at the nonzero value $[23,28]$.

The nonzero VEVs break $\mathrm{SU}(N) \times \mathrm{SU}\left(N_{F}\right)_{F} \times \mathrm{U}(1)_{\phi}$ gauge symmetry to $\mathrm{SU}(\tilde{N}) \times$ $\mathrm{SU}(N)_{D} \times \mathrm{U}(1)_{Y} \cdot \mathrm{SU}(N)_{D}$ and $\mathrm{U}(1)_{Y}$ are the linear combinations of the subgroups of $\mathrm{SU}\left(N_{F}\right)_{F}$ and $\mathrm{SU}(N) \times \mathrm{U}(1)_{\phi}$.

\subsection{Gauge bosons}

After the symmetry breaking, massive gauge bosons appear according to the Higgs mechanism. Let us decompose the vector field $\left(V_{F}^{\mu}\right)$ for $\mathrm{SU}\left(N_{F}\right)_{F}$ as

$$
V_{F}^{\mu}=\left(\begin{array}{cc}
W_{F}^{\mu}-a B^{\prime \mu} & \frac{1}{\sqrt{2}}\left(X^{\mu}\right)^{\dagger} \\
\frac{1}{\sqrt{2}} X^{\mu} & G^{\mu}+\frac{N}{\widetilde{N}} a B^{\prime \mu}
\end{array}\right),
$$

where $a=\frac{\sqrt{\widetilde{N}}}{\sqrt{2 N(N+\widetilde{N})}}$ is defined. $\quad W_{F}^{\mu}$ and $G^{\mu}$ are the adjoint representations of the subgroups of $\mathrm{SU}\left(N_{F}\right)_{F}: \mathrm{SU}(N)_{F}$ and $\mathrm{SU}(\widetilde{N}) . X_{\mu}$ is the anti-fundamental and fundamental representations of $\mathrm{SU}(N)_{F} \times \mathrm{SU}(\widetilde{N})$, and $B_{\mu}^{\prime}$ is the $\mathrm{U}(1)_{F}$ vector field, where $\mathrm{U}(1)_{F}$ is from $\mathrm{SU}\left(N_{F}\right)_{F}$.

The nonzero VEVs generate the following mass terms,

$$
\begin{aligned}
\mathcal{L}_{g} & =M_{X}^{2} X_{\mu}^{\dagger} X^{\mu}+\frac{1}{2} M_{W^{\prime}}^{2} W_{\mu}^{\prime A} W^{\prime A \mu}+\frac{1}{2} M_{Z^{\prime}}^{2} Z_{\mu}^{\prime} Z^{\prime \mu}, \\
M_{X}^{2} & =g_{F}^{2}\left(v_{\chi}^{2}+\Delta v^{2}\right), \\
M_{W^{\prime}}^{2} & =2\left(g_{F}^{2}+g_{N}^{\prime 2}\right) v_{\chi}^{2}, \\
M_{Z^{\prime}}^{2} & =4 N\left(Q_{\phi}^{2} g_{\phi}^{2}+a^{2} g_{F}^{2}\right) v_{\chi}^{2},
\end{aligned}
$$




\begin{tabular}{|c|cccc|ccc|c|}
\hline & $Z$ & $\widetilde{Z}$ & $\rho$ & $\widetilde{\rho}$ & $Y$ & $\chi$ & $\widetilde{\chi}$ & $X$ \\
\hline $\mathrm{SU}(\widetilde{N})$ & $\widetilde{\mathbf{N}}$ & $\widetilde{\mathbf{N}}$ & $\widetilde{\mathbf{N}}$ & $\widetilde{\widetilde{N}}$ & $\mathbf{1}$ & $\mathbf{1}$ & $\mathbf{1}$ & $\operatorname{adj}_{\widetilde{N}}$ \\
$\mathrm{SU}(N)_{D}$ & $\overline{\mathbf{N}}$ & $\mathbf{N}$ & $\overline{\mathbf{N}}$ & $\mathbf{N}$ & $\operatorname{adj}_{N}$ & $\operatorname{adj}_{N}$ & $\operatorname{adj}_{N}$ & $\mathbf{1}$ \\
$\mathrm{U}(1)_{Y}$ & $\frac{N+\widetilde{N}}{N \widetilde{N}}$ & $\frac{-N-\widetilde{N}}{N \widetilde{N}}$ & $\frac{N+\widetilde{N}}{N \widetilde{N}}$ & $\frac{-N-\widetilde{N}}{N \widetilde{N}}$ & 0 & 0 & 0 & 0 \\
\hline
\end{tabular}

Table 2. Extra Chiral superfields charged under the $\mathrm{SU}(\tilde{N}) \times \mathrm{SU}(N)_{D} \times \mathrm{U}(1)_{Y}$.

where $\Delta v=v_{X}-v_{Y}$ is defined. $W^{\prime A \mu}$ and $Z^{\prime \mu}$ are given by the linear combinations of $W_{F}^{A \mu}$ and $\mathrm{SU}(N)$ gauge boson $\left(W_{N}^{A \mu}\right)$, and $B^{\prime \mu}$ and $\mathrm{U}(1)_{\phi}$ gauge boson $\left(A_{\phi}^{\mu}\right)$ respectively:

$$
\begin{aligned}
\left(\begin{array}{c}
B^{\prime \mu} \\
A_{\phi}^{\mu}
\end{array}\right) & =\left(\begin{array}{cc}
\cos \theta_{Y} & \sin \theta_{Y} \\
-\sin \theta_{Y} & \cos \theta_{Y}
\end{array}\right)\left(\begin{array}{c}
B^{\mu} \\
Z^{\prime \mu}
\end{array}\right), \\
\left(\begin{array}{c}
W_{F}^{A \mu} \\
W_{N}^{A \mu}
\end{array}\right) & =\left(\begin{array}{cc}
\cos \theta & -\sin \theta \\
\sin \theta & \cos \theta
\end{array}\right)\left(\begin{array}{c}
W^{A \mu} \\
W^{\prime A \mu}
\end{array}\right),
\end{aligned}
$$

where $\cos \theta_{Y}$ and $\cos \theta$ are defined as

$$
\cos \theta_{Y}=\frac{Q_{\phi} g_{\phi}}{\sqrt{Q_{\phi}^{2} g_{\phi}^{2}+a^{2} g_{F}^{2}}}, \quad \cos \theta=\frac{g_{N}^{\prime}}{\sqrt{g_{N}^{\prime 2}+g_{F}^{2}}} .
$$

$G_{\mu}, W_{\mu}$, and $B_{\mu}$ are the gauge bosons for $\mathrm{SU}(\tilde{N}) \times \mathrm{SU}(N)_{D} \times \mathrm{U}(1)_{Y}$ gauge symmetry, and their gauge couplings are given by

$$
g_{N}=g_{F} \cos \theta, g_{\widetilde{N}}=g_{F}, g_{1}^{\prime}=a N g_{1}=a N g_{F} \cos \theta_{Y} .
$$

\subsection{SM-charged fields from symmetry breaking sector}

According to the decomposition in eqs. (2.4) and (2.5), we introduce the charge assignment of $(Z, \widetilde{Z}),(\rho, \widetilde{\rho}), Y,(\chi, \widetilde{\chi})$, and $X$ in table $2 . Y,(\chi, \widetilde{\chi})$, and $X$ are the adjoint parts of $\hat{Y},(\hat{\chi}, \hat{\tilde{\chi}})$, and $\hat{X}$. The singlet parts are not charged under the SM, and they are not so relevant to our analysis. The mass matrices are studied in appendix A.

These fields obtain masses according to the nonzero VEVs, $v_{\chi}, v_{Y}$ and $v_{X}$ as we see in the appendix A. They decouple at some scales above the EW scale. In the next subsection, we investigate the RG flows of the gauge couplings including the threshold corrections and discuss the soft SUSY breaking terms mediated by the heavy fields.

\subsection{RG flows of the gauge couplings}

In this model, two kinds of symmetry breaking actually happen: one is $\mathrm{SU}\left(N_{F}\right)_{F}$ breaking, $\mathrm{SU}\left(N_{F}\right)_{F} \rightarrow \mathrm{SU}(\widetilde{N}) \times \mathrm{SU}(N)_{F} \times \mathrm{U}(1)_{F}$, and the other is $\mathrm{SU}(N)_{F} \times \mathrm{U}(1)_{F} \times \mathrm{SU}(N) \times \mathrm{U}(1)_{\phi}$ breaking: $\mathrm{SU}(N)_{F} \times \mathrm{SU}(N) \rightarrow \mathrm{SU}(N)_{D}$ and $\mathrm{U}(1)_{F} \times \mathrm{U}(1)_{\phi} \rightarrow \mathrm{U}(1)_{Y}$. The former is caused by $\Delta v$, and the later is by $v_{\chi}$. We consider a simple scenario assuming $\Delta v \gg v_{\chi}$.

As we see in appendix A, there will be several intermediate scales, where heavy particles in the symmetry breaking sector are decoupled and the RG flow of gauge couplings is 
modify. According to the one-loop RG equations, the gauge couplings at the EW scale $\left(M_{Z}\right)$ are evaluated as follows: $\mathrm{SU}(N)_{F}, \mathrm{SU}(N)$ and $\mathrm{SU}(N)_{D}$ gauge couplings $\left(\alpha_{F_{N}}, \alpha_{N}^{\prime}, \alpha_{N}\right)$ are

$$
\begin{aligned}
& 4 \pi \alpha_{N}^{-1}\left(M_{Z}\right)=4 \pi \alpha_{F_{N}}^{-1}\left(T_{\chi_{N}}\right)+4 \pi \alpha_{N}^{\prime-1}\left(T_{\chi_{N}}\right)+b_{N} \ln \left(\frac{M_{Z}^{2}}{T_{\chi_{N}}^{2}}\right)+\Delta b_{\mathrm{ex}}^{N}\left(\frac{T_{\mathrm{ex}}^{2}}{\Lambda^{2}}\right), \\
& 4 \pi \alpha_{F_{N}}^{-1}\left(T_{\chi_{N}}\right)=4 \pi \alpha_{G}^{-1}(\Lambda)+\Delta b_{N} \ln \left(\frac{T_{N}^{2}}{\Lambda^{2}}\right)+\left(b_{N}-2 N\right) \ln \left(\frac{T_{\chi_{N}}^{2}}{\Lambda^{2}}\right) \\
& 4 \pi \alpha_{N}^{\prime-1}\left(T_{\chi_{N}}\right)=4 \pi \alpha_{N}^{\prime-1}(\Lambda)+\Delta b_{\rho_{N}} \ln \left(\frac{T_{\rho_{N}}^{2}}{\Lambda^{2}}\right)-N \ln \left(\frac{T_{\chi_{N}}^{2}}{\Lambda^{2}}\right) .
\end{aligned}
$$

$\mathrm{SU}(\widetilde{N})$ gauge coupling $\left(\alpha_{\widetilde{N}}\right)$ is

$$
\begin{aligned}
4 \pi \alpha_{\widetilde{N}}^{-1}\left(M_{Z}\right)= & 4 \pi \alpha_{G}^{-1}(\Lambda)+b_{\widetilde{N}} \ln \left(\frac{M_{Z}^{2}}{\Lambda^{2}}\right)+\Delta b_{\widetilde{N}} \ln \left(\frac{T_{\widetilde{N}}^{2}}{\Lambda^{2}}\right) \\
& +\Delta b_{\mathrm{eX}}^{\widetilde{N}} \ln \left(\frac{T_{\mathrm{ex}}^{2}}{\Lambda^{2}}\right)+\Delta b_{\rho_{\widetilde{N}}} \ln \left(\frac{T_{\rho_{\widetilde{N}}^{2}}^{2}}{\Lambda^{2}}\right)+\Delta b_{X} \ln \left(\frac{T_{\chi_{\widetilde{N}}}^{2}}{\Lambda^{2}}\right) .
\end{aligned}
$$

$\mathrm{U}(1)_{F}, \mathrm{U}(1)_{\phi}$, and $\mathrm{U}(1)_{Y}$ gauge couplings $\left(\alpha_{F_{1}}, \alpha_{\phi}, \alpha_{1}\right)$ are

$$
\begin{aligned}
& 4 \pi \alpha_{1}^{-1}\left(M_{Z}\right)=4 \pi \alpha_{F_{1}}^{-1}\left(T_{\chi_{1}}\right)+\frac{4 \pi a^{2}}{Q_{\phi}^{2}} \alpha_{\phi}^{-1}\left(T_{\chi_{1}}\right)+b_{1} \ln \left(\frac{M_{Z}^{2}}{T_{\chi_{1}}^{2}}\right)+\Delta b_{\mathrm{ex}}^{1} \ln \left(\frac{T_{\mathrm{ex}}^{2}}{\Lambda^{2}}\right), \\
& 4 \pi \alpha_{F_{1}}^{-1}\left(T_{\chi_{1}}\right)=4 \pi \alpha_{G}^{-1}(\Lambda)+\Delta b_{1} \ln \left(\frac{T_{1}^{2}}{\Lambda^{2}}\right) !+\Delta b_{\rho_{1}} \ln \left(\frac{T_{\rho_{1}}^{2}}{\Lambda^{2}}\right)+\left(b_{1}+\Delta b_{\chi_{1}}\right) \ln \left(\frac{T_{\chi_{1}}^{2}}{\Lambda^{2}}\right), \\
& 4 \pi \alpha_{\phi}^{-1}\left(T_{\chi_{1}}\right)=4 \pi \alpha_{\phi}^{-1}(\Lambda)+\Delta b_{\rho_{\phi}} \ln \left(\frac{T_{\rho_{1}}^{2}}{\Lambda^{2}}\right)+\Delta b_{\chi_{\phi}} \ln \left(\frac{T_{\chi_{1}}^{2}}{\Lambda^{2}}\right) .
\end{aligned}
$$

$\Lambda$ is the cut-off scale and $T_{i}, T_{\chi_{i}}$ and $T_{\rho_{i}}(i=N, \widetilde{N}, 1)$ are the intermediate scales where $X_{\mu}, \chi_{i}\left(\chi_{\widetilde{N}} \equiv X\right)$, and $\rho_{i}$ decouple respecitvely. According to the mass spectrums at each scale in appendix $\mathrm{A}, T_{i}, T_{\chi_{i}}$ and $T_{\rho_{i}}(i=N, \widetilde{N}, 1)$ are estimated as

$$
\begin{aligned}
\left(T_{N}, T_{\rho_{N}}, T_{\chi_{N}}\right) & =\left(M_{X}, h \Delta v, \sqrt{h} M_{G^{\prime}}\right), \\
\left(T_{\widetilde{N}}, T_{\rho_{\widetilde{N}}}, T_{\chi_{\widetilde{N}}}\right) & =\left(M_{X}, h \Delta v, m_{X}\right), \\
\left(T_{1}, T_{\rho_{1}}, T_{\chi_{1}}\right) & =\left(M_{X}, h \Delta v, \sqrt{h} M_{Z^{\prime}}\right) .
\end{aligned}
$$

The factor in front of each intermediate scale describes the freedom of the particles decoupling at the scale:

$$
\begin{aligned}
\left(\Delta b_{N}, \Delta b_{\rho_{N}}\right) & =\left(2\left(N_{F}-N\right),-\widetilde{N}\right), \\
\left(\Delta b_{\widetilde{N}}, \Delta b_{\rho_{\tilde{N}}}, \Delta b_{X}\right) & =\left(2\left(N_{F}-\widetilde{N}\right),-N,-\widetilde{N}\right), \\
\left(\Delta b_{1}, \Delta b_{\rho_{1}}, \Delta b_{\chi_{1}}\right) & =\left(2 N_{F},-a^{2} N^{2} \frac{2 N}{\widetilde{N}},-2 a^{2} N^{2}\right), \\
\left(\Delta b_{\rho_{\phi}}, \Delta b_{\chi_{\phi}}\right) & =\left(-2 N \widetilde{N} Q_{\phi}^{2},-2 N^{2} Q_{\phi}^{2}\right) .
\end{aligned}
$$


We may also have to introduce additional particles charged under the gauge symmetry, in order to achieve realistic mass spectrums. For instance, colored Higgs would be necessary to derive the MSSM Higgs doublet at the low scale in section 3, and it is charged under $\mathrm{SU}(\widetilde{N}) \times \mathrm{U}(1)_{F}$ in our explicit model. Such an extra intermediate scale and the coefficient is defined as $T_{\text {ex }}$ and $\Delta b_{\text {ex }}^{J}(J=\widetilde{N}, 1)$.

We also study the soft SUSY breaking terms of sfermions in the next subsection. Let us also introduce the wave function renormalization factor $\left(Z_{q}\right)$ for $\mathrm{SU}(N)_{F}$-charged field $(q)$. The one-loop renormalization group for $Z_{q}$ can be integrated analytically, if the Yukawa coupling is negligible,

$$
\begin{aligned}
\ln Z_{q}\left(M_{Z}\right)= & \ln Z_{q}(\Lambda)+\frac{2 c_{G}^{q}}{b_{G}} \ln \left(\frac{\alpha_{G}(\Lambda)}{\alpha_{G}\left(T_{i}\right)}\right)+\frac{2 c_{i}^{q}}{b_{G}-\Delta b_{i}} \ln \left(\frac{\alpha_{F_{i}}\left(T_{i}\right)}{\alpha_{F_{i}}\left(T_{\mathrm{ex}}\right)}\right) \\
& +\frac{2 c_{i}^{q}}{b_{G}-\Delta b_{i}-\Delta b_{\mathrm{ex}}^{i}} \ln \left(\frac{\alpha_{F_{i}}\left(T_{\mathrm{ex}}\right)}{\alpha_{F_{i}}\left(T_{\rho_{i}}\right)}\right) \\
& +\frac{2 c_{i}^{q}}{b_{G}-\Delta b_{i}-\widehat{\Delta b_{\rho_{i}}}-\Delta b_{\mathrm{ex}}^{i}} \ln \left(\frac{\alpha_{F_{i}}\left(T_{\rho_{i}}\right)}{\alpha_{F_{i}}\left(T_{\chi_{i}}\right)}\right)+\frac{2 c_{i}^{q}}{b_{i}} \ln \left(\frac{\alpha_{i}\left(T_{\chi_{i}}\right)}{\alpha_{i}\left(M_{Z}\right)}\right)
\end{aligned}
$$

where $\left(\widetilde{\Delta b_{\rho_{N}}}, \widetilde{\Delta b_{\rho_{\tilde{N}}}}, \widetilde{\Delta b_{\rho_{1}}}\right)=\left(0, \Delta b_{\rho_{\tilde{N}}}, \Delta b_{\rho_{1}}\right)$ is defined and $T_{i} \geq T_{\mathrm{ex}} \geq T_{\rho_{i}}$ is assumed. $c_{G}^{q}$ and $c_{i}^{q}$ are the second Casimir of the field $q$, corresponding to the gauge groups. The masses squared of sfermions can be derived by the $v_{X}$-dependence in $Z_{q} \cdot v_{X}$ appears in the gauge couplings, so that $v_{X}$-dependence on the gauge couplings is only relevant to the sfermion masses [29].

\subsection{Soft SUSY breaking terms}

Based on the above results, we investigate soft SUSY breaking terms which relate to particles charged under the gauge symmetry. Soft SUSY breaking terms in $\mathrm{SU}(\widetilde{N}) \times \operatorname{SU}(N)_{D} \times$ $\mathrm{U}(1)$ are calculated by substituting $v_{X}+\theta^{2} F_{X}$ for $v_{X}$ in the gauge couplings [29]. Compared to typical gauge mediation, where messengers are only chiral superfields, massive gauge bosons and the fermionic partners also work as the mediators to generate the soft SUSY breaking terms, in our models [24, 25, 30, 31].

In eqs. $(2.17),(2.20)$, and (2.21), the only intermediate scales, $T_{i}, T_{\rho_{i}}$, and $T_{\mathrm{ex}}$ depend on $v_{X}$. This leads the masses $\left(M_{\widetilde{N}}, M_{N}, M_{1}\right)$ of the gauginos, which are the superpartner of $\mathrm{SU}(N)_{D} \times \mathrm{SU}(\widetilde{N}) \times \mathrm{U}(1)$ gauge bosons, as follows:

$$
\begin{aligned}
& M_{N}(\mu)=-\left(\Delta b_{N}+\Delta b_{\rho_{N}}+\Delta b_{\mathrm{ex}}^{N} \xi_{N}\right) \frac{\alpha_{N}(\mu)}{4 \pi} \frac{F_{X}}{|\Delta v|}, \\
& M_{\widetilde{N}}(\mu)=-\left(\Delta b_{\widetilde{N}}+\Delta b_{\rho_{\widetilde{N}}}+\Delta b_{\mathrm{ex}}^{\widetilde{N}} \xi_{\widetilde{N}}\right) \frac{\alpha_{\widetilde{N}}(\mu)}{4 \pi} \frac{F_{X}}{|\Delta v|}, \\
& M_{1}(\mu)=-\left(\Delta b_{1}+\Delta b_{\rho_{1}}+\frac{a^{2}}{Q_{\phi}^{2}} \Delta b_{\rho_{\phi}}+\Delta b_{\mathrm{ex}}^{1} \xi_{1}\right) \frac{\alpha_{1}(\mu)}{4 \pi} \frac{F_{X}}{|\Delta v|} .
\end{aligned}
$$

$\xi_{N}, \xi_{\widetilde{N}}$, and $\xi_{1}$ describe the $v_{X}$ dependence on the mass scale of extra particles, $T_{\mathrm{ex}}$. For example, the holomorphic mass of extra particles may be given by $m_{\mathrm{ex}}+\lambda_{\mathrm{ex}}\left(v_{X}+\theta^{2} F_{X}\right)$, where $m_{\mathrm{ex}}$ and $\lambda_{\mathrm{ex}}$ are a supersymmetric mass term and Yukawa coupling involving the 
extra particles. That is, the gaugino mass contribution of $\ln \left(T_{\text {ex }}\right)$ would be proportional to $\lambda_{\mathrm{ex}} F_{X} / m_{\mathrm{ex}}$, if $m_{\mathrm{ex}}$ is larger than $\lambda_{\mathrm{ex}} v_{X}$. In this case, $\xi_{i}$ is approximately given by $\xi_{i}=\lambda_{\mathrm{ex}}|\Delta v| / m_{\mathrm{ex}}$.

Let us consider the soft SUSY breaking terms corresponding to the trilinear (A-term) and bilinear couplings (B-term) of the scalar components of the $\mathrm{SU}\left(N_{F}\right)_{F}$-charged fields $\left(q_{I}\right)$. They are relevant to the $v_{X}$-dependence of the wave renormalization factor. For instance, the A-terms corresponding to the Yukawa couplings $y_{I J K} q_{I} q_{J} q_{K}$ in the superpotential are given by $A_{I J K}=A_{I}+A_{J}+A_{K}$, where $A_{I}=\frac{\partial \ln Z_{I}}{\partial \ln v_{X}}$ is defined and the trilinear coupling is described as $y_{I J K} A_{I J K} q_{I} q_{J} q_{K}$.

Eventually, $A_{I}$ is obtained from eq. (2.31),

$$
\begin{aligned}
A_{I}= & \left\{2 c_{G}^{I} \frac{\alpha_{G}\left(T_{i}\right)}{4 \pi}-\frac{2 b_{G} c_{i}^{I}}{b_{G}-\Delta b_{i}} \frac{\alpha_{F_{i}}\left(T_{i}\right)}{4 \pi}\right. \\
& +\left(\frac{2 c_{i}^{I}}{b_{G}-\Delta b_{i}}-\frac{2 c_{i}^{I}}{b_{G}-\Delta b_{i}-\Delta b_{\mathrm{ex}}^{i}}\right)\left(b_{G} \xi+\Delta b_{i}(1-\xi)\right) \frac{\alpha_{F_{i}}\left(T_{\mathrm{ex}}\right)}{4 \pi} \\
& +\left(\frac{2 c_{i}^{I}}{b_{G}-\Delta b_{i}-\Delta b_{\mathrm{ex}}^{i}}-\frac{2 c_{i}^{I}}{b_{G}-\Delta b_{i}-\Delta b_{\mathrm{ex}}^{i}-\widetilde{\Delta b_{\rho_{i}}}}\right)\left(b_{G}-\Delta b_{\mathrm{ex}}^{i}(1-\xi)\right) \frac{\alpha_{F_{i}}\left(T_{\rho_{i}}\right)}{4 \pi} \\
& +\left(\frac{2\left(\Delta b_{i}+\Delta b_{\mathrm{ex}}^{i} \xi+\widetilde{\Delta b_{\rho_{i}}}\right) c_{i}^{I}}{b_{G}-\Delta b_{i}-\Delta b_{\mathrm{ex}}^{i}-\widetilde{\Delta b_{\rho_{i}}}}\right) \frac{\alpha_{F_{i}}\left(T_{\chi_{i}}\right)}{4 \pi}-\left(\frac{2\left(\Delta b_{i}+\Delta b_{\mathrm{ex}}^{i} \xi+\Delta b_{\rho_{i}}^{\prime}\right) c_{i}^{I}}{b_{i}}\right) \frac{\alpha_{i}\left(T_{\chi_{i}}\right)}{4 \pi} \\
& \left.+\left(\frac{2\left(\Delta b_{i}+\Delta b_{\mathrm{ex}}^{i} \xi+\Delta b_{\rho_{i}}^{\prime}\right) c_{i}^{I}}{b_{i}}\right) \frac{\alpha_{i}(\mu)}{4 \pi}\right\} \frac{F_{X}}{|\Delta v|}
\end{aligned}
$$

assuming $\xi_{N}=\xi_{\widetilde{N}}=\xi_{1}=\xi \cdot \alpha_{F_{\tilde{N}}} \equiv \alpha_{\widetilde{N}}$ is defined.

The masses squared $\left(m_{q}^{2}\right)$ of $q$ could be also estimated by the eq. (2.31), seeing the $\left|v_{X}\right|^{2}$ dependence of $Z_{q}$ [29]. As discussed in ref. [30], the gauge mediation with gauge messengers may contribute to the masses squared at the one-loop level, if the gauge symmetry breaking and SUSY breaking are caused by the VEVs and F-components of several fields. In our case, we simply assume $v_{\chi} \ll \Delta v$, so that the gauge symmetry breaking and SUSY breaking are caused by only $\Delta v$ and the F-component of $\Delta v .^{3}$ The one-loop correction is strongly suppressed by $\left(v_{\chi} / \Delta v\right)^{2}$ according to ref. [30], so that we have to investigate the two-loop corrections, as discussed in refs. [24, 29].

Following refs. $[24,29], m_{q}^{2}$ could be written as

$$
\begin{aligned}
m_{q}^{2}(\mu)= & \left\{2 b_{G} c_{G}^{q} \frac{\alpha_{G}^{2}\left(T_{i}\right)}{(4 \pi)^{2}}-\frac{2 b_{G}^{2} c_{i}^{q}}{b_{G}-\Delta b_{i}} \frac{\alpha_{F_{i}}^{2}\left(T_{i}\right)}{(4 \pi)^{2}}\right. \\
& +\left(\frac{2 c_{i}^{q}}{b_{G}-\Delta b_{i}}-\frac{2 c_{i}^{q}}{b_{G}-\Delta b_{i}-\Delta b_{\mathrm{ex}}^{i}}\right)\left(b_{G} \xi+\Delta b_{i}(1-\xi)\right)^{2} \frac{\alpha_{F_{i}}^{2}\left(T_{\mathrm{ex}}\right)}{(4 \pi)^{2}} \\
& +\left(\frac{2 c_{i}^{q}}{b_{G}-\Delta b_{i}-\Delta b_{\mathrm{ex}}^{i}}-\frac{2 c_{i}^{q}}{b_{G}-\Delta b_{i}-\Delta b_{\mathrm{ex}}^{i}-\widetilde{\Delta b_{\rho_{i}}}}\right)\left(b_{G}-\Delta b_{\mathrm{ex}}^{i}(1-\xi)\right)^{2} \frac{\alpha_{F_{i}}^{2}\left(T_{\rho_{i}}\right)}{(4 \pi)^{2}}
\end{aligned}
$$

\footnotetext{
${ }^{3} \Delta v$ corresponds to the $\mathrm{VEV}$ of one adjoint field.
} 


$$
\begin{aligned}
& +\left(\frac{2\left(\Delta b_{i}+\Delta b_{\mathrm{ex}}^{i} \xi+\widetilde{\Delta b_{\rho_{i}}}\right)^{2} c_{i}^{q}}{b_{G}-\Delta b_{i}-\Delta b_{\mathrm{ex}}^{i}-\widetilde{\Delta b_{\rho_{i}}}}\right) \frac{\alpha_{F_{i}}^{2}\left(T_{\chi_{i}}\right)}{(4 \pi)^{2}} \\
& -\left(\frac{2\left(\Delta b_{i}+\Delta b_{\mathrm{ex}}^{i} \xi+\Delta b_{\rho_{i}}^{\prime}\right)^{2} c_{i}^{q}}{b_{i}}\right) \frac{\alpha_{i}^{2}\left(T_{\chi_{i}}\right)}{(4 \pi)^{2}} \\
& \left.+\left(\frac{2\left(\Delta b_{i}+\Delta b_{\mathrm{ex}}^{i} \xi+\Delta b_{\rho_{i}}^{\prime}\right)^{2} c_{i}^{q}}{b_{i}}\right) \frac{\alpha_{i}^{2}(\mu)}{(4 \pi)^{2}}\right\} \frac{F_{X}^{2}}{|\Delta v|^{2}},
\end{aligned}
$$

where $\Delta b_{\rho_{i}}^{\prime}$ is $\left(\Delta b_{\rho_{3}}^{\prime}, \Delta b_{\rho_{2}}^{\prime}, \Delta b_{\rho_{1}}^{\prime}\right)=\left(\Delta b_{\rho_{3}}, \Delta b_{\rho_{2}}, \Delta b_{\rho_{1}}+a^{2} \Delta b_{\rho_{\phi}} / Q_{\phi}^{2}\right)$.

In the next section, we discuss one explicit model, where $\mathrm{SU}(\tilde{N}) \times \mathrm{SU}(N)_{D} \times \mathrm{U}(1)_{Y}$ is the SM gauge groups corresponding to $\left(N_{F}, N\right)=(5,2)$. In the explicit model, we see that a few parameters control all soft SUSY breaking terms according to this analysis. Then, $\Lambda_{\text {SUSY }}$ is roughly given by $\left(\alpha_{G} /(4 \pi)\right) \times\left(F_{X} /|\Delta v|\right)$, and A-term and B-term are of $O\left(\Lambda_{\mathrm{SUSY}}\right)$, which we could expect that are consistent with the condition for the EW symmetry breaking. We study the compatibility with the EW condition and the Higgs mass, in section 3.4 .

\section{$3 \mathrm{SU}(5)_{F} \times \mathrm{SU}(2) \times \mathrm{U}(1)_{\phi}$ gauge theory: $\left(N_{F}, N\right)=(5,2)$}

In this section, we consider a $\mathrm{SU}(5)_{F} \times \mathrm{SU}(2) \times \mathrm{U}(1)_{\phi}$ gauge symmetric model, which correspond to the $\left(N_{F}, N\right)=(5,2)$ case. We expect that the MSSM fields are embedded in to $\mathbf{1 0}$ and $\overline{\mathbf{5}}$ representation as in the Georgi-Glashow SU(5) GUT. Involving $\mathbf{5}$-representation Higgs $(H, \bar{H})$, the superpotential for the Yukawa couplings in the visible sector is

$$
W_{v i s}=\hat{y}_{k l}^{u} H \mathbf{1 0} \mathbf{1}^{k} \mathbf{0}^{l}+\hat{y}_{k l}^{d} \bar{H} \overline{\mathbf{5}}^{k} \mathbf{1 0} \mathbf{0}^{l}
$$

where $\overline{\mathbf{5}}^{k}$ and $\mathbf{1 0} \mathbf{0}^{l}$ are defined as the matter fields. As well-known, $\hat{y}_{k l}^{u}$ and $\hat{y}_{k l}^{d}$ may require $\Phi$ and $(\phi, \widetilde{\phi})$ dependences in order to generate realistic mass matrices at the EW scale according to the higher-dimensional operators. Here, we simply assume that the contributions to the soft SUSY breaking terms are enough small.

One serious problem in the SU(5) GUT is how to generate the mass splitting between the colored Higgs and the MSSM Higgs doublet. The mass of colored Higgs should be around the GUT scale to avoid the too short life time of proton: $m_{H_{c}} \gtrsim$ $10^{16} \mathrm{GeV} \times\left(1 \mathrm{TeV} / \Lambda_{\mathrm{SUSY}}\right)[32,33]$. In our $\mathrm{SU}(5)_{F} \times \mathrm{SU}(2) \times \mathrm{U}(1)_{\phi}$ model, the relevant terms to the Higgs masses is written as

$$
W_{H}=\mu \bar{H} H+\lambda_{H} \bar{H} \Phi H .
$$

After the symmetry breaking, the colored Higgs mass and MSSM Higgs mass are given by $\mu+\lambda v_{X}$ and $\mu+\lambda_{H} v_{Y}$. If $v_{Y}=m_{\phi} / h$ is the GUT scale, $\mu$ should be also around the GUT scale and then the fine-tuning between $\mu$ and $\lambda v_{Y}$ is required: $\mu+\lambda_{H} v_{Y} \approx O\left(M_{Z}\right)$. On the other hand, we could expect that the colored Higgs is enough heavy because of $\mu$, if there is no cancellation between $\mu$ and $\lambda_{H} v_{X}$. Let us also consider the case that $v_{X}$ is the GUT scale. In this case, the MSSM Higgs mass could be light if $\mu$ and $v_{Y}$ are around the weak scale, and the colored Higgs is heavy: $m_{H_{c}} \approx \lambda_{H} v_{X}$. 


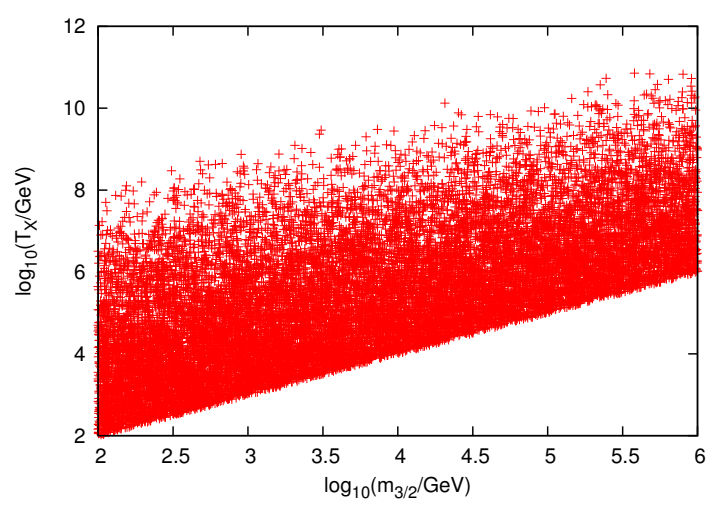

Figure 1. Gravitino mass $\left(m_{3 / 2}\right)$ and the scale $T_{X}$ with $10^{16} \mathrm{GeV} \leq T_{G} \leq M_{p} . T_{X}$ should be small to rase the GUT scale above $10^{16} \mathrm{GeV}$. $T_{\chi}$ is $T_{\chi} \approx \sqrt{M_{p} m_{3 / 2}}$. The constraints, $T_{\rho}>T_{\chi}$ and $T_{X}>m_{3 / 2}$, are also assigned. All gauge couplings and Yukawa couplings satisfy the perturbative bounds as $\alpha_{F_{i}}<4 \pi$.

In both cases, the colored Higgs couples with $v_{X}+F_{X} \theta^{2}$, so it mediates the SUSY breaking effect to the soft SUSY breaking terms. The supersymmetric mass for $\mathrm{SU}(2)_{L}$ Higgs doublet is $\mu_{2}=\mu+\lambda_{H} v_{Y} \approx O\left(M_{Z}\right)$. On the other hand, the colored-Higgs mass is $m_{H_{c}}=\mu+\lambda_{H} v_{X} \approx \lambda_{H}\left(v_{Y}-v_{X}\right)$, so that $\xi$ for the colored Higgs in soft SUSY breaking terms is approximately estimated as $\xi \approx \operatorname{sign}\left(\lambda_{H}\right)$. The one-loop correction of $H_{c}$ to $m_{q}^{2}$ would be suppressed, because the $m_{H_{c}}$-dependence appears in $Z_{q}$ as $\ln \left(\left|m_{H_{c}}+\lambda_{H} F_{X} \theta^{2}\right|^{2}\right)$ according to the study in ref. [29]. We could apply our analysis in section 2.4 to this scenario.

\subsection{Gauge couplings}

In this model, $\mathrm{SU}(5)_{F} \times \mathrm{SU}(2) \times \mathrm{U}(1)_{\phi}$ breaks down to the $\mathrm{SM}$ gauge group, $\mathrm{SU}(3)_{c} \times$ $\mathrm{SU}(2)_{L} \times \mathrm{U}(1)_{Y} . \mathrm{SU}(3)_{c}$ is the subgroup of $\mathrm{SU}(5)_{F}$ and $\mathrm{SU}(2)_{L} \times \mathrm{U}(1)_{Y}$ are the linear combination of $\mathrm{SU}(2)_{F} \times \mathrm{U}(1)_{F}$ and $\mathrm{SU}(2) \times \mathrm{U}(1)_{\phi}$.

On the other hand, there are several intermediate scales: $\left(T_{G}, T_{\rho}, T_{\chi}, T_{X}\right){ }^{4} T_{G}$ is the GUT scale, where $X_{\mu}$ decouples, and $T_{\rho}$ is the messenger scale fixed by the parameter $h$ and the GUT scale. $T_{\chi}$ is interpreted as the SUSY breaking scale, because $T_{\chi} \approx \sqrt{F_{X}}=$ $\sqrt{M_{p} m_{3 / 2}}$, so that it is almost fixed around $O\left(10^{10}\right) \mathrm{GeV}$ when $m_{3 / 2}=O(100) \mathrm{GeV} . T_{X}$ is fixed by the mass scale of $X\left(m_{X}\right)$, which is massless at the tree-level. $X$ could be expected to be $O\left(\Lambda_{\mathrm{SUSY}}\right)$, because the one-loop corrections shift the mass, but it may be difficult to clearly fix the masses of bosonic and fermonic $X$ in our model. Let us simply treat $m_{X}$ as the free parameter, and figure 1 shows the allowed region for $T_{X}$, which may not be far from $O\left(\Lambda_{\mathrm{SUSY}}\right)$. Figure 2 shows the gauge couplings, $\left(\alpha_{F 2}, \alpha_{2}, \alpha_{F 1}, \alpha_{\phi}\right)$ at the SUSY breaking scale. Figure 3 describes RG flows of the gauge couplings $\left(\alpha_{3}, \alpha_{2}\left(\alpha_{F_{2}}\right), \alpha_{1}\left(\alpha_{F_{1}}\right)\right)$, when $T_{X}=10^{7} \mathrm{GeV}, T_{\chi}=3.8 \times 10^{10} \mathrm{GeV}, T_{\rho}=7.9 \times 10^{11} \mathrm{GeV}$, and $T_{\mathrm{GUT}}=2 \times 10^{16} \mathrm{GeV}$.

\footnotetext{
${ }^{4} T_{\chi_{1}}=T_{\chi_{2}}, T_{\rho_{1}}=T_{\rho_{3}}$, and $T_{G}=T_{1}=T_{2}=T_{3}$ are assumed.
} 

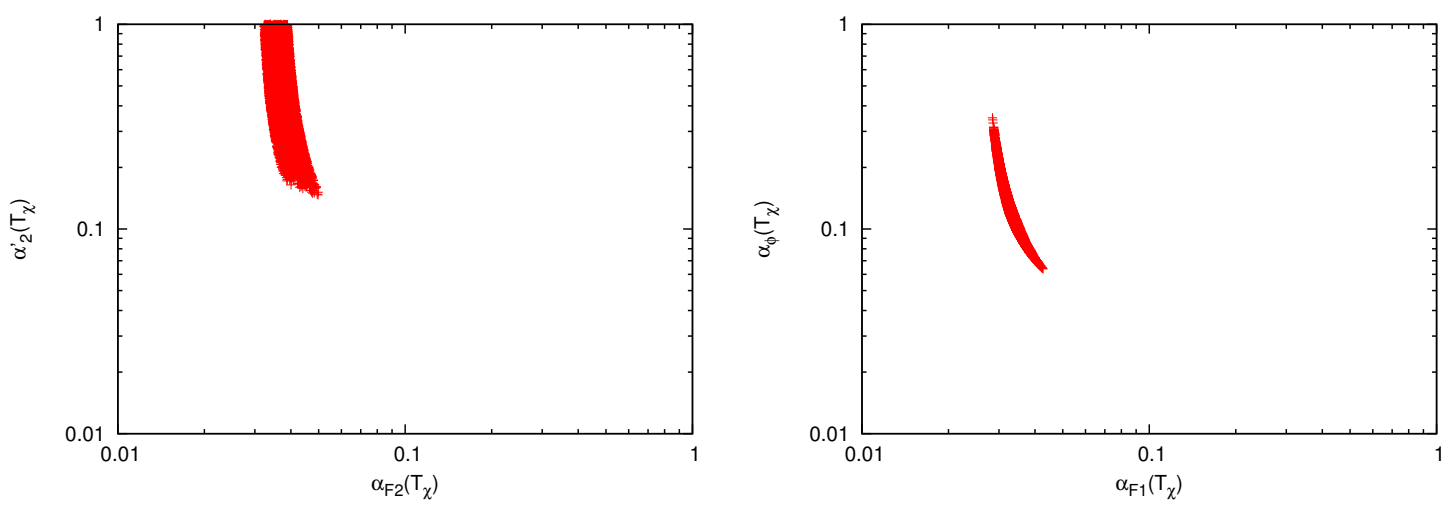

Figure 2. $\alpha_{F_{2}}$ vs. $\alpha_{2}^{\prime}$ and $\alpha_{F_{1}}$ vs. $\alpha_{\phi}$ at the symmetry breaking scale, $T_{\chi}$.

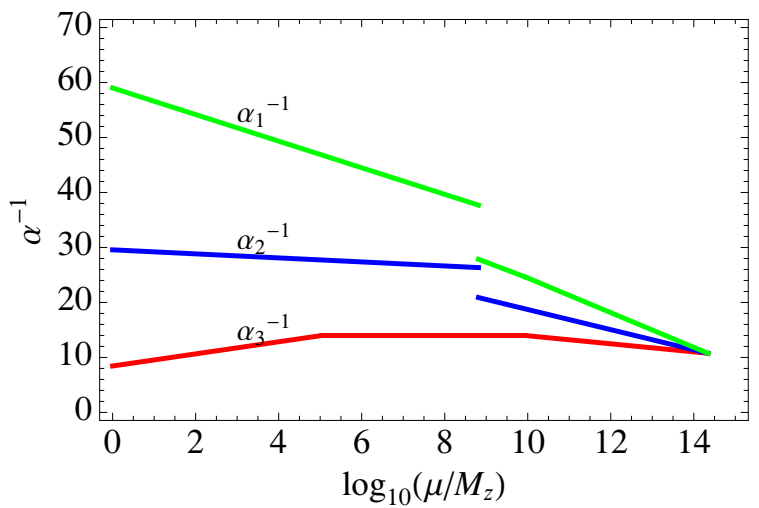

Figure 3. RG flows of the subgroups of $\mathrm{SU}(5)_{F}$, with $T_{X}=10^{7} \mathrm{GeV}, T_{\chi}=3.8 \times 10^{10} \mathrm{GeV}$, $T_{\rho}=7.9 \times 10^{11} \mathrm{GeV}$, and $T_{\mathrm{GUT}}=2 \times 10^{16} \mathrm{GeV}$. The green, blue, and red lines correspond to the gauge couplings of $\mathrm{U}(1)_{Y} \times \mathrm{SU}(2)_{L} \times \mathrm{SU}(3)_{c}$ below $T_{\chi}$ and $\mathrm{U}(1)_{F} \times \mathrm{SU}(2)_{F} \times \mathrm{SU}(3)_{c}$ above $T_{\chi}$ respectively. The input parameters for the couplings are in eq. (3.6).

\subsection{Soft SUSY breaking terms}

We qualitatively evaluate the soft SUSY breaking terms in this scenario. According to the analysis in section 2.4 , the gaugino masses at $\mu<T_{\chi}$ are written as

$$
\begin{aligned}
& M_{2}(\mu)=-3 \frac{\alpha_{2}(\mu)}{4 \pi} \frac{F_{X}}{|\Delta v|}, \\
& M_{3}(\mu)=-(2-\xi) \frac{\alpha_{3}(\mu)}{4 \pi} \frac{F_{X}}{|\Delta v|}, \\
& M_{1}(\mu)=-\left(\frac{37-2 \xi}{5}\right) \frac{\alpha_{1}(\mu)}{4 \pi} \frac{F_{X}}{|\Delta v|} .
\end{aligned}
$$

Let us consider the case with $\xi=1$ and the gaugino masses at the EW scale. The gauge couplings at the EW scale are [3]

$$
\alpha_{1}\left(M_{Z}\right) \approx 0.01695, \alpha_{2}\left(M_{Z}\right) \approx 0.03382, \alpha_{3}\left(M_{Z}\right) \approx 0.1185
$$


so that we could derive the following mass relation:

$$
\frac{M_{1}\left(M_{Z}\right)}{M_{3}\left(M_{Z}\right)} \approx 1.001, \frac{M_{2}\left(M_{Z}\right)}{M_{3}\left(M_{Z}\right)} \approx 0.856
$$

The masses are almost degenerate, and this may be a specific feature of the gauge messenger model $[24,34] .{ }^{5}$ If all intermediate scales are close to the GUT scale, the fine-tuning of $\mu$ term may be drastically reduced, as discussed in ref. [36]. Figure 2 tells us that the extra $\mathrm{SU}(3)$-adjoint field reside in the low-scale, so that the condition for the small $\mu$-term would be modified. The one-loop running correction of $m_{H_{u}}^{2}$ with $T_{X}=10^{7} \mathrm{GeV}$ from $T_{\chi}$ to $M_{Z}$ is estimated as

$$
\Delta m_{H_{u}}^{2} \approx-0.276 M_{3}\left(M_{Z}\right)^{2}-0.047 M_{2}\left(M_{Z}\right) M_{3}\left(M_{Z}\right)+0.221 M_{2}\left(M_{Z}\right)^{2}+\ldots,
$$

where the ellipsis denotes the terms including A-term and scalar masses and those are not important when they are comparable to the gluino mass. This leads that the condition to cancel the large contribution of gluino is $M_{2} / M_{3}\left(M_{Z}\right) \approx 1.23$, which suggests the almost degenerate mass spectrum. However, we have a large A-term contribution to $\Delta m_{H_{u}}^{2}$ in our model, so that it may be difficult to avoid a certain fine-tuning even if the gaugino masses are degenerate.

According to eqs. (2.36) and (2.35), the masses squared of superpartners and A-term are evaluated explicitly. Setting $T_{G}=T_{H_{c}}>T_{\rho}>T_{\chi}$ and $\xi=1$, stop masses at $T_{\chi}$ are given by

$$
\begin{aligned}
& m_{Q}^{2}\left(T_{\chi}\right) \approx\left(8.83-6.67 \frac{\alpha_{3}^{2}\left(T_{\rho}\right)}{\alpha_{G}^{2}}-10.80 \frac{\alpha_{F_{2}}^{2}\left(T_{\chi}\right)}{\alpha_{G}^{2}}-0.33 \frac{\alpha_{F_{1}}^{2}\left(T_{\chi}\right)}{\alpha_{G}^{2}}\right) \Lambda_{\mathrm{SUSY}}^{2}, \\
& m_{U}^{2}\left(T_{\chi}\right) \approx\left(8.60-6.67 \frac{\alpha_{3}^{2}\left(T_{\rho}\right)}{\alpha_{G}^{2}}-5.30 \frac{\alpha_{F_{1}}^{2}\left(T_{\chi}\right)}{\alpha_{G}^{2}}-0.01 \frac{\alpha_{F_{1}}^{2}\left(T_{\rho}\right)}{\alpha_{G}^{2}}\right) \Lambda_{\mathrm{SUSY}}^{2} .
\end{aligned}
$$

As we see, large stop masses are generated by the large second casimir $\left(c_{2}^{t}=18 / 5\right)$, but they might be driven to the tachyonic if $T_{\chi}$ and $T_{\rho}$ are close to the GUT scale. The SUSY scale $\left(\Lambda_{\mathrm{SUSY}}\right)$ from the gauge mediation is defined as

$$
\Lambda_{\mathrm{SUSY}}=\frac{\alpha_{G}}{(4 \pi)} \frac{F_{X}}{|\Delta v|} \approx \frac{\alpha_{G}}{(4 \pi)} \frac{M_{p}}{T_{G}} m_{3 / 2} .
$$

$\alpha_{G}$ is of $O(0.1)$ when $T_{G}$ is around $10^{16} \mathrm{GeV}$, so that $\Lambda_{\text {SUSY }}$ might be compatible with $m_{3 / 2}$. If $T_{G}$ is smaller, the situation, $\Lambda_{\mathrm{SUSY}} \gg m_{3 / 2}$, is achieved but suffers from the constraint from proton decay. The correction from the gravity mediation is naively estimated as $O\left(m_{3 / 2}\right)$. It is almost the same order as the one from the gauge mediation in our model, and it may make it difficult to control flavors. In fact, the gauge-mediation contributions are typically at least 5 times as large as the gravitino mass in our model, as we see in table 4 . In this case, we could expect the gravity-mediation effect is sub-dominant, and the SUSY scale is governed by the gauge-mediation. However, the gravity-mediation contribution

\footnotetext{
${ }^{5}$ The gaugino masses are degenerate in the TeV-scale mirage mediation scenario, too [35].
} 
should be $O\left(10^{-2}\right)$ times suppressed, if it contributes to the sparticles masses squared flavor-universally [37]. In order to realize such a suppression and control flavor in the MSSM, we have to consider flavor symmetry or some dynamics above the GUT scale, as discussed in refs. [38-44]. ${ }^{6}$ Indeed, explicit contributions on soft masses through the gravity mediation depend on the UV completion of our model. In this letter, one of our main motivations is to achieve $125 \mathrm{GeV}$ Higgs mass and realistic EW symmetry breaking, which may be independent of this issue about the constraint from flavor physics, so that we will discuss our SUSY mass spectrums assuming that the gauge-mediation is dominant. The underlying theory above the GUT scale will be studied in ref. [55].

$A_{t}$, which is the trilinear coupling of stops $(\widetilde{t})$ as $y_{t} A_{t} \widetilde{t}_{L} H_{u} \widetilde{t}_{R}$ is given by

$$
A_{t}\left(T_{\chi}\right) \approx\left(22.57-8.00 \frac{\alpha_{3}\left(T_{\rho}\right)}{\alpha_{G}}-3.6 \frac{\alpha_{F_{2}}\left(T_{\chi}\right)}{\alpha_{G}}-0.98 \frac{\alpha_{F_{1}}\left(T_{\chi}\right)}{\alpha_{G}}+0.01 \frac{\alpha_{F_{1}}\left(T_{\rho}\right)}{\alpha_{G}}\right) \Lambda_{\mathrm{SUSY}}
$$

and the B-term, which is the bilinear coupling of two Higgs $\mu B H_{u} H_{d}$, is estimated as

$$
B\left(T_{\chi}\right) \approx\left(10.27-3.60 \frac{\alpha_{F_{2}}\left(T_{\chi}\right)}{\alpha_{G}}-0.68 \frac{\alpha_{F_{1}}\left(T_{\chi}\right)}{\alpha_{G}}+0.01 \frac{\alpha_{F_{1}}\left(T_{\rho}\right)}{\alpha_{G}}\right) \Lambda_{\mathrm{SUSY}} .
$$

As we see, the A-term and B-term might be large as $O(10) \Lambda_{\mathrm{SUSY}}$. This may be good to achieve the EW symmetry breaking, but too large A-term makes the stop masses tachyonic because of the running correction such as

$$
\Delta m_{U}^{2}\left(M_{Z}\right) \approx-0.08 A_{t}\left(T_{\chi}\right)^{2}+1.54 M_{3}\left(T_{\chi}\right)^{2}-0.15 A_{t}\left(T_{\chi}\right) M_{3}\left(T_{\chi}\right) .
$$

In our model, the gluino mass $M_{3}$ is relatively small as wee see in eq. (3.4), so $\Delta m_{U}^{2}\left(M_{Z}\right)$ becomes easily negative and stop mass becomes tachyonic even if the positive $m_{U}^{2}$ is generated at the SUSY breaking scale $T_{\chi}$. In order to avoid the tachyonic stop masses, we add an extra contribution to the gluino mass, as we see below.

\subsection{Shift of the gluino mass}

We consider an extra term, which contributes to the gluino mass,

$$
W=\frac{1}{\Lambda_{0}} \operatorname{Tr}_{5}\left(\Phi W_{5} W_{5}\right)
$$

There are several ways to introduce this term, such as gravity effect. Here, we simply assume that $N_{\text {extra }}$ extra heavy SU(5) vector-like pairs $(\psi, \bar{\psi})$ with the masses $\bar{\psi}\left(\Lambda_{0}+\lambda_{X} \Phi\right) \psi$ induce this term, integrating out them at the scale $\Lambda_{0}$. After the $\mathrm{SU}(5)$ breaking, the gauge coupling would have the extra $v_{X}$ dependence as

$$
\alpha_{3}^{-1} \rightarrow \alpha_{3}^{-1}-\frac{N_{\text {extra }}}{4 \pi} \ln \left(\frac{\left(\left|\Lambda_{0}+\lambda_{X}\left(v_{X}+F_{X} \theta^{2}\right)\right|^{2}\right)}{\Lambda^{2}}\right) .
$$

This additional coupling could shift the gluino mass as

$$
M_{3} \rightarrow M_{3}-\frac{\alpha_{3} N_{\mathrm{eff}}}{4 \pi} \frac{F_{X}}{|\Delta v|}
$$

\footnotetext{
${ }^{6}$ In fact, such strong dynamics has been proposed not only to suppress flavor changing currents but also to realize the superpotential $W_{\mathrm{SB}}$ in section $2[26]$.
} 

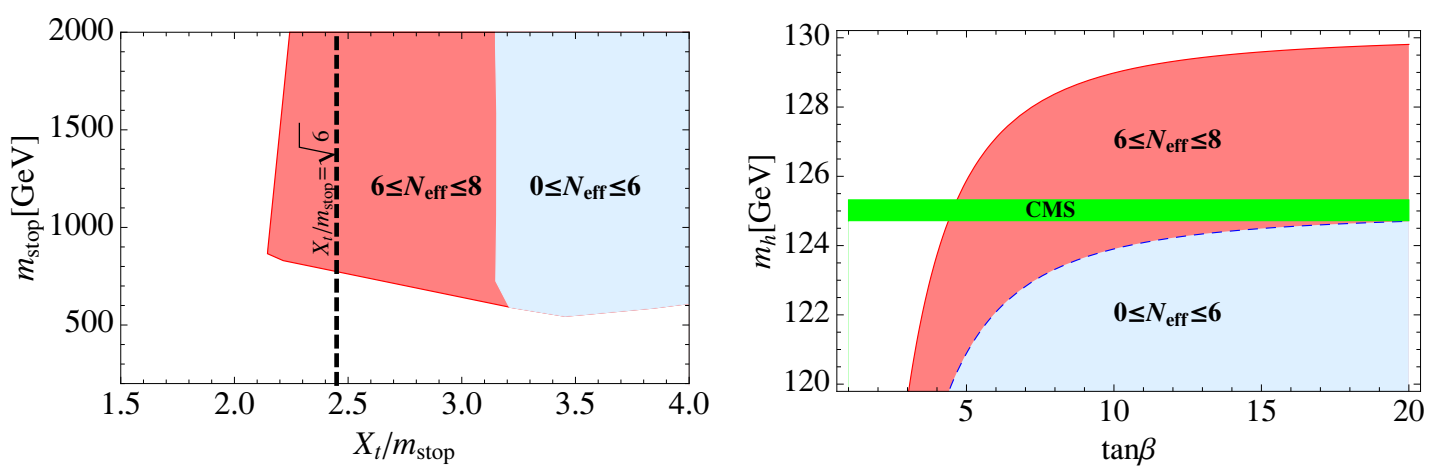

Figure 4. $X_{t} / m_{\text {stop }}$ vs. $m_{\text {stop }}$ and $\tan \beta$ vs. the lightest Higgs mass in the case with $\left(T_{\mathrm{GUT}}, T_{X}\right)=$ $\left(2 \times 10^{16} \mathrm{GeV}, 10^{7} \mathrm{GeV}\right)$ and $0 \leq N_{\text {eff }} \leq 6$ (light blue), $6 \leq N_{\text {eff }} \leq 8$ (light red). The dashed line corresponds to $X_{t} / m_{\text {stop }}=\sqrt{6}$. In the right figure, $m_{h}$ is calculated at the two-loop level using $m_{t}=172.9 \mathrm{GeV}$, and $m_{\text {stop }}$ is lighter than $2 \mathrm{TeV}$. The green band is the CMS result on Higgs mass from $h \rightarrow \gamma \gamma, Z Z$ channels [47].

where $N_{\text {eff }}$ may not be $N_{\text {extra }}$ because of the scale difference between $\Lambda_{0}$ and the GUT scale. Including $N_{\text {eff }}$, the gluino mass becomes

$$
M_{3}(\mu)=-\left(1-N_{\mathrm{eff}}\right) \frac{\alpha_{3}(\mu)}{4 \pi} \frac{F_{X}}{|\Delta v|},
$$

so $N_{\text {eff }}$ should be bigger than 2 in order to shift $M_{3}$. In fact, we discuss large $N_{\text {eff }}$ cases and find that $N_{\text {eff }}$ enables us to evade the negative squared masses and achieve the large SM Higgs mass.

\subsection{Consistency with the Higgs mass and the EW symmetry breaking}

One issue in supersymmetric models is how to realize the $\mu$ and $B$ terms which are consistent with the EW scale. Especially, $\mu$ relates to the lightest Higgs mass, because of the upper bound in MSSM, so that the recent Higgs discovery with the mass $125 \mathrm{GeV}$ may impose unnatural SUSY scenarios on us. In fact, $125 \mathrm{GeV}$ Higgs mass may require $\Lambda_{\mathrm{SUSY}} \gtrsim O(10) \mathrm{TeV}$ in the simple scenarios as discussed in refs. [4, 5]. $O(10)-\mathrm{TeV}$ SUSY scale would require $0.01 \%$ fine-tuning against $\mu$ without any cancellation in $m_{H_{u}}^{2}$. As pointed out in refs. $[45,46]$, it is known that a special relation between $A_{t}$ and squark mass relaxes the fine-tuning, maximizing the loop corrections in the Higgs mass in the MSSM. This relation is so-called "maximal mixing" and described as $X_{t} / m_{\text {stop }}=\sqrt{6}$, where $X_{t}=A_{t}-\mu / \tan \beta$ and $m_{\text {stop }}^{2}=\sqrt{m_{Q}^{2} m_{U}^{2}}$ are defined. If this relation is satisfied, the $125 \mathrm{GeV}$ Higgs mass could be achieved even if the stop is light. We can see our prediction on $X_{t}$ and the upper bound on the Higgs mass in the case with $0 \leq N_{\text {eff }} \leq 6$ (light blue), $6 \leq N_{\text {eff }} \leq 8$ (light red) in figure 4 . On the all regions, all masses squared of the superpartners are positive and $\left(T_{G}, T_{X}\right)$ are fixed at $\left(2 \times 10^{16} \mathrm{GeV}, 10^{7} \mathrm{GeV}\right)$. We find that our A-term is too large to realize $X_{t} / m_{\text {stop }}=\sqrt{6}$, but the maximal mixing could be achieved, if we allow large $N_{\text {eff }}$, and enhance the Higgs mass, even if $m_{\text {stop }}$ is around $1 \mathrm{TeV}$.

On the other hand, we notice that there is no special cancellation in $m_{H_{u}}^{2}$ and $m_{H_{d}}^{2}$, as we see in figure 5 . Large $m_{\text {stop }}$ corresponds to large $\mu$, so that $1-\mathrm{TeV}$ squark mass requires 

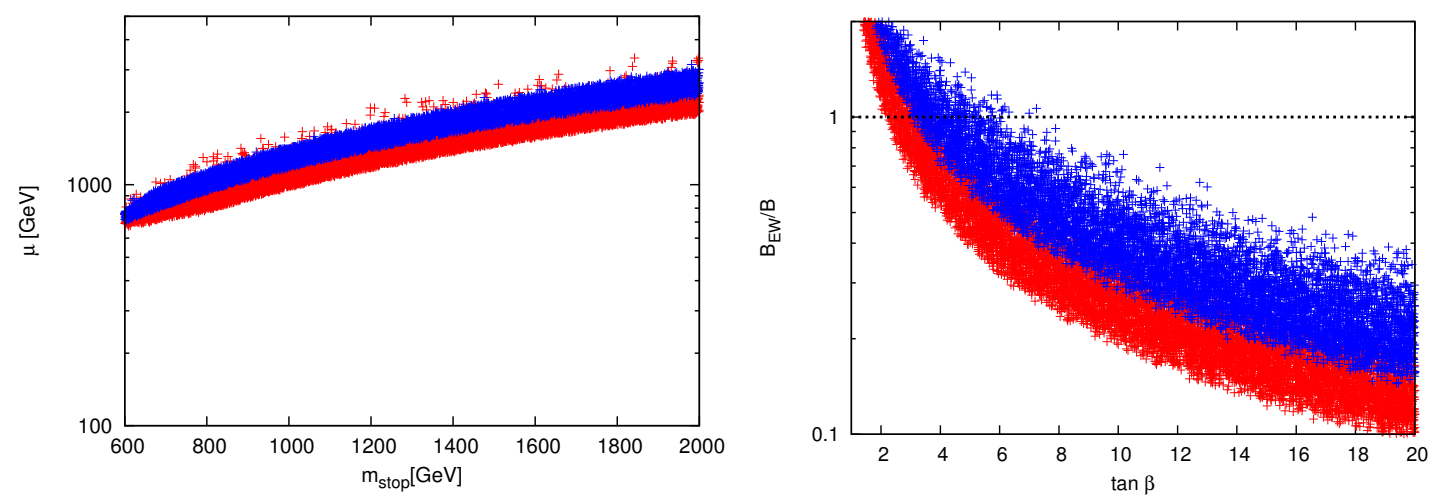

Figure 5. $m_{\text {stop }}$ vs. $\mu$ and $\tan \beta$ vs. B-term in the case with $\left(T_{\mathrm{GUT}}, T_{X}\right)=\left(2 \times 10^{16} \mathrm{GeV}, 10^{7} \mathrm{GeV}\right)$ and $0 \leq N_{\text {eff }} \leq$ (blue), $6 \leq N_{\text {eff }} \leq 8$ (red). In the right figure, $m_{\text {stop }}$ is lighter than $2 \mathrm{TeV}$. The dashed line is consistent with the condition for the EW symmetry breaking.

$1 \%$ fine-tuning against $\mu$. The right figure in figure 5 shows that small $\tan \beta$ is consistent with the EW symmetry breaking. $B_{\mathrm{EW}}$ is the value to realize the EW symmetry breaking,

$$
B_{\mathrm{EW}} \equiv-\frac{1}{2 \mu}\left\{\left(m_{H_{d}}^{2}-m_{H_{u}}^{2}\right) \tan 2 \beta+M_{Z}^{2} \sin 2 \beta\right\},
$$

and $B$ is our prediction via the gauge mediation. It seems that $2 \lesssim \tan \beta \lesssim 6$ is necessary to achieve $125 \mathrm{GeV}$ Higgs mass. The $\tan \beta$ region may be inconsistent with the one required by $125 \mathrm{GeV}$ Higgs $(\tan \beta \gtrsim 4)$ with $m_{\text {stop }} \leq 2 \mathrm{TeV}$. Table 4 in appendix $\mathrm{B}$ shows the parameter sets in our model, which satisfy $m_{h} \approx 125 \mathrm{GeV}$ and $\left|B_{\mathrm{EW}} / B\right| \approx 1$. There, $m_{\text {stop }}$ and $|\mu|$ are around $3 \mathrm{TeV}$, and $O(0.1) \%$ fine-tuning is required against $\mu$ term.

\section{$4 \quad \mathrm{SU}(5)_{F} \times \mathrm{SU}(3) \times \mathrm{U}(1)_{\phi}$ gauge theory: $\left(N_{F}, N\right)=(5,3)$}

Our symmetry breaking model could be embed into other type GUT model. One simple example would be the $\mathrm{SU}(5)_{F} \times \mathrm{SU}(3) \times \mathrm{U}(1)_{\phi}$ gauge symmetric model, and we could consider the same setup as in the $\mathrm{SU}(5)_{F} \times \mathrm{SU}(2) \times \mathrm{U}(1)_{\phi}$ gauge theory. The visible sector is given by eq. (3.1). However, the modification of the Higgs sector may be required because $\lambda \bar{H} \Phi H$ term gives the very large $\mathrm{B}$-term, $\lambda F_{X} H_{u} H_{d}$. There may be a solution to realize the EW symmetry breaking, but the serious fine-tuning may be required. Here, we consider another solution to shift the colored Higgs mass which maybe favor high-scale SUSY.

We introduce $\mathrm{SU}(3)$ vector-like fields $\left(H_{3}, \bar{H}_{3}\right)$ and assign $Z_{3}$ symmetry to the fields as in table $3 . Z_{3}$ symmetry is broken by the VEV of $S$. The superpotential for the Higgs sector is given by

$$
W_{H}=\lambda_{S} S \bar{H} H+\lambda_{\phi} \bar{H}_{3} \phi+\lambda_{\widetilde{\phi}} \widetilde{\phi} H_{3} H+\frac{\lambda_{3}}{3} S^{3} .
$$

After the GUT symmetry breaking, $(H, \bar{H})$ are decomposed as $\left(\left(H_{u}, H_{3}^{\prime}\right),\left(H_{d}, \bar{H}_{3}^{\prime}\right)\right)$ and the mass terms for $\left(H_{3}, \bar{H}_{3}^{\prime}\right)$ and $\left(H_{3}^{\prime}, \bar{H}_{3}\right)$ pairs appear as

$$
W_{H}^{\text {eff }}=\lambda_{\phi} v_{\chi} \bar{H}_{3}^{\prime} \bar{H}_{3}+\lambda_{\widetilde{\phi}} v_{\chi} H_{3} H_{3}^{\prime} .
$$




\begin{tabular}{|c|cccc|ccc|ccc|}
\hline & $\overline{\mathbf{5}}_{i}$ & $\mathbf{1 0}_{i}$ & $H$ & $\bar{H}$ & $H_{3}$ & $\bar{H}_{3}$ & $S$ & $\phi$ & $\widetilde{\phi}$ & $\Phi$ \\
\hline $\mathrm{SU}(5)_{F}$ & $\overline{\mathbf{5}}$ & $\mathbf{1 0}$ & $\mathbf{5}$ & $\overline{\mathbf{5}}$ & $\mathbf{1}$ & $\mathbf{1}$ & $\mathbf{1}$ & $\mathbf{5}$ & $\overline{\mathbf{5}}$ & $\mathbf{a d j}_{5}+\mathbf{1}$ \\
$\mathrm{SU}(3)$ & $\mathbf{1}$ & $\mathbf{1}$ & $\mathbf{1}$ & $\mathbf{1}$ & $\mathbf{3}$ & $\overline{\mathbf{3}}$ & $\mathbf{1}$ & $\mathbf{3}$ & $\overline{\mathbf{3}}$ & $\mathbf{1}$ \\
$\mathrm{U}(1)_{\phi}$ & 0 & 0 & 0 & 0 & $Q_{\phi}$ & $-Q_{\phi}$ & 0 & $Q_{\phi}$ & $-Q_{\phi}$ & 0 \\
\hline$Z_{3}$ & $\omega$ & $\omega$ & $\omega$ & $\omega$ & $\omega^{2}$ & $\omega^{2}$ & $\omega$ & $\mathbf{1}$ & $\mathbf{1}$ & $\mathbf{1}$ \\
\hline
\end{tabular}

Table 3. Chiral superfields in $\mathrm{SU}(5)_{F} \times \mathrm{SU}(3) \times \mathrm{U}(1)$ gauge theory.

$H_{u}$ and $H_{d}$ correspond to the Higgs $\mathrm{SU}(2)_{L}$ doublets in MSSM, and they could get the supersymmetric mass term according to the nonzero VEV of $S$. In refs. [48-54], we can see not only the $\mathrm{SU}(5)_{F} \times \mathrm{SU}(2) \times \mathrm{U}(1)_{\phi}$-type but also this type of product-GUT.

In order to avoid the bound from the proton decay caused by the five dimensional operators, $v_{\chi}$ should be large as

$$
v_{\chi} \gtrsim 10^{16} \mathrm{GeV} \times\left(\frac{1 \mathrm{TeV}}{\Lambda_{\mathrm{SUSY}}}\right) .
$$

$F_{X}$ is given by $-h v_{\chi}^{2}$, so that very tiny $h$ is necessary to achieve the low-scale SUSY. When $v_{\chi} \approx 10^{16} \mathrm{GeV}$ and $\Lambda_{\mathrm{SUSY}}=1 \mathrm{TeV}$ are set, $h$ should be around $O\left(10^{-10}\right)$, because of

$$
h=\frac{4 \pi}{\alpha_{G}} \frac{\Delta v}{v_{\chi}^{2}} \Lambda_{\mathrm{SUSY}} \approx 10^{-10} \times\left(\frac{10^{16} \mathrm{GeV}}{v_{\chi}}\right)^{2}\left(\frac{\Lambda_{\mathrm{SUSY}}}{1 \mathrm{TeV}}\right) \lesssim 10^{-10} \times\left(\frac{\Lambda_{\mathrm{SUSY}}}{1 \mathrm{TeV}}\right)^{3} .
$$

We conclude that high-scale SUSY is favored to avoid such an extremely small $h$.

We can consider the applications of our symmetry breaking models to the other BSMs, such as

- $\mathrm{SU}(3)_{c} \times \mathrm{SU}(2)_{L} \times \mathrm{SU}(2)_{R} \times \mathrm{U}(1)_{B-L} \rightarrow \mathrm{SU}(3)_{c} \times \mathrm{SU}(2)_{L} \times \mathrm{U}(1)_{Y}$,

- $\mathrm{SU}(4) \times \mathrm{SU}(2)_{L} \times \mathrm{U}(1) \rightarrow \mathrm{SU}(3)_{c} \times \mathrm{SU}(2)_{L} \times \mathrm{U}(1)_{Y}$.

We would study such patterns elsewhere [55]. In these models, all of chiral superfields appear as adjoint representations and bi-fundamental representations. Such models can be constructed in D-brane models, e.g. intersecting/magnetized D-brane models (see for a review $[56,57]$ and references therein). Thus, the above models are interesting from the viewpoint of superstring theory.

\section{Summary}

The MSSM is one of the attractive BSMs to solve the hierarchy problem in the SM and it may be expected to be found near future. One big issue in the MSSM is how to control the SUSY breaking parameters, so that many ideas and works on spontaneous SUSY breaking and mediation mechanisms of the SUSY breaking effects have been discussed so far. In this paper, we proposed an explicit and simple supersymmetric model, where the spontaneous SUSY breaking and GUT breaking are achieved by the same sector. The origin of the 
hyper-charge assignment in the MSSM is also explained by the analogy with the GeorgiGlashow SU(5) GUT. The SM-charged particles are also introduced by the breaking sector, so that we could also predict the soft SUSY breaking terms via the gauge mediation with the gauge and chiral messenger superfields. The crucial role of the gauge-messenger mediation is to induce large A-terms and B-terms at the one-loop level. We investigated the scenario with light superpartners that such a large A-term realizes the maximal mixing and shift the lightest Higgs mass. In fact, we have to introduce additional contribution to the gluino mass, but $125 \mathrm{GeV}$ Higgs mass could be achieved, even if stop is light. $m_{\text {stop }}$ should be as light as possible to relax the fine-tuning of $\mu$ parameter. On the other hand, the oneloop B-term could be also consistent with the EW symmetry breaking, if $\tan \beta$ is within $2 \lesssim \tan \beta \lesssim 6$. Such small $\tan \beta$ may require large stop mass, as we see in figures 4 and 5. In fact, we see that about $3 \mathrm{TeV} m_{\text {stop }}$ can achieve $125 \mathrm{GeV}$ Higgs mass and the EW symmetry breaking in table 4 .

Our light SUSY particles are wino, bino, and gravitino, and the mass difference is not so big. The lightest particle is bino, and wino is heavier than bino. The mass difference is $O(0.1) \times m_{3 / 2} \mathrm{GeV}$. This might be one specific feature of the gauge messenger scenario in $\mathrm{SU}(5) \mathrm{GUT}$, as discussed in ref. [34].

\section{Acknowledgments}

We are grateful to Hiroyuki Abe for useful discussions and comments. This work is supported by Grant-in-Aid for Scientific research from the Ministry of Education, Science, Sports, and Culture (MEXT), Japan, N0. 25400252 (T.K.) and No. 23104011 (Y.O.).

\section{A Mass spectrums of the particles in the symmetry breaking sector}

We investigate the mass matrices for the remnant fields in the symmetry breaking sector. First, let us discuss $(Z, \widetilde{Z})$ and $(\rho, \widetilde{\rho})$ components. We define $Z_{ \pm}$and $\rho_{ \pm}$as

$$
Z_{ \pm}=\frac{\widetilde{Z} \pm Z^{\dagger}}{\sqrt{2}}, \rho_{ \pm}=\frac{\rho \pm \widetilde{\rho}^{\dagger}}{\sqrt{2}}
$$

The fermion masses are given by

$$
\mathcal{L}_{f}=-\left(\overline{\lambda_{-}} \overline{Z_{+}} \overline{\rho_{+}}\right) M_{+}^{f}\left(\begin{array}{c}
\lambda_{-} \\
Z_{+} \\
\rho_{+}
\end{array}\right)-\left(\overline{\lambda_{+}} \overline{Z_{-}} \overline{\rho_{-}}\right) M_{-}^{f}\left(\begin{array}{c}
\lambda_{+} \\
Z_{-} \\
\rho_{-}
\end{array}\right)
$$

where the mass matrices $\left(M_{ \pm}^{f}\right)$ are

$$
M_{+}^{f}=\left(\begin{array}{ccc}
0 & -g \Delta v & g v_{\chi} \\
-g \Delta v & 0 & -h v_{\chi} \\
g v_{\chi} & -h v_{\chi} & -h \Delta v
\end{array}\right), M_{-}^{f}=\left(\begin{array}{ccc}
0 & -g \Delta v & g v_{\chi} \\
-g \Delta v & 0 & h v_{\chi} \\
g v_{\chi} & h v_{\chi} & h \Delta v
\end{array}\right)
$$


and $\lambda_{ \pm}$are the linear combinations of the gauginos $\left(X_{(+)}\right)$which are the suparpartners of $X_{\mu}$,

$$
\lambda_{ \pm}=\frac{X_{+} \pm \bar{X}}{\sqrt{2}}
$$

The masses for the bosonic superpartners are

$$
\mathcal{L}_{B}=-\left(\begin{array}{ll}
Z_{+}^{\dagger} & \rho_{+}^{\dagger}
\end{array}\right) M_{+}^{2}\left(\begin{array}{c}
Z_{+} \\
\rho_{+}
\end{array}\right)-\left(Z_{-}^{\dagger} \rho_{-}^{\dagger}\right) M_{-}^{2}\left(\begin{array}{c}
Z_{-} \\
\rho_{-}
\end{array}\right)
$$

where the mass matrices $\left(M_{ \pm}^{2}\right)$ are given by

$$
\begin{aligned}
& M_{+}^{2}=\left(\begin{array}{cc}
h^{2} v_{\chi}^{2} & -h^{2} v_{\chi} \Delta v \\
-h^{2} v_{\chi} \Delta v & h^{2}\left(v_{\chi}^{2}+\Delta v^{2}\right)+F_{X}
\end{array}\right), \\
& M_{-}^{2}=\left(\begin{array}{cc}
h^{2} v_{\chi}^{2}+g^{2} \Delta v^{2} & -\left(h^{2}+g^{2}\right) \Delta v v_{\chi} \\
-\left(h^{2}+g^{2}\right) \Delta v v_{\chi} & h^{2}\left(v_{\chi}^{2}+\Delta v^{2}\right)+g^{2} v_{\chi}^{2}-F_{X}
\end{array}\right) .
\end{aligned}
$$

The F-term $F_{X}$ is $F_{X}=-h^{2} v_{\chi}^{2}$, so that $M_{+}^{2}$ includes the Goldstone mode.

The fermion masses for the other particles are also generated by the VEVs:

$$
\mathcal{L}_{Y}=-\frac{1}{2}\left(\widetilde{W}^{A} Y^{A}\right) M_{Y}\left(\begin{array}{c}
\chi^{A} \\
\widetilde{\chi}^{A}
\end{array}\right)-\frac{1}{2}\left(\begin{array}{ll}
\chi^{A} & \widetilde{\chi}^{A}
\end{array}\right) M_{Y}^{T}\left(\begin{array}{c}
\widetilde{W}^{A} \\
Y^{A}
\end{array}\right)+\text { h.c. },
$$

where $\widetilde{W}$ is the superpartner of $W^{\prime}$ and $M_{Y}$ are defined as

$$
M_{Y}=\left(\begin{array}{cc}
-\frac{1}{\sqrt{2}} M_{W^{\prime}} & \frac{1}{\sqrt{2}} M_{W^{\prime}} \\
-h v_{\chi} & -h v_{\chi}
\end{array}\right) .
$$

The eigenvalues are $M_{W^{\prime}}, M_{W^{\prime}}, \sqrt{2} h v_{\chi}, \sqrt{2} h v_{\chi}$ and the bosonic masses are given by the same mass spectrum. The imaginary part of $\chi-\tilde{\chi}$ corresponds to the Goldstone boson, and the real part has the mass, $M_{W^{\prime}}$, according to the D-term. The other masses, $\sqrt{2} h v_{\chi}$, correspond to the ones of $\chi+\widetilde{\chi}$ and $Y$.

The singlet components $\left(Y_{0}, \chi_{0}, \tilde{\chi}_{0}\right)$ of $\hat{Y}$ and $(\hat{\chi}, \hat{\tilde{\chi}})$ also get masses, according to the nonzero $v_{\chi}$. The fermionic mass matrix is

$$
\mathcal{L}_{Y_{0}}=-\frac{1}{2}\left(\widetilde{Z^{\prime}} Y_{0}\right) M_{Y_{0}}\left(\begin{array}{l}
\chi_{0} \\
\widetilde{\chi_{0}}
\end{array}\right)-\frac{1}{2}\left(\begin{array}{ll}
\chi_{0} & \widetilde{\chi_{0}}
\end{array}\right) M_{Y_{0}}^{T}\left(\begin{array}{c}
\widetilde{Z^{\prime}} \\
Y_{0}
\end{array}\right)+h . c .,
$$

where $M_{Y_{0}}$ are defined as

$$
M_{Y_{0}}=\left(\begin{array}{cc}
-\frac{1}{\sqrt{2}} M_{Z^{\prime}} & \frac{1}{\sqrt{2}} M_{Z^{\prime}} \\
-h v_{\chi} & -h v_{\chi}
\end{array}\right)
$$

The mass spectrums are given, relplacing $M_{W^{\prime}}$ with $M_{Z^{\prime}}$. 


\begin{tabular}{|c|c|c|c|c|}
\hline & $N_{\mathrm{eff}}=6$ & $N_{\text {eff }}=6$ & $N_{\text {eff }}=6.97$ & $N_{\text {eff }}=7.83$ \\
\hline$m_{3 / 2}$ & $588.84 \mathrm{GeV}$ & $741.31 \mathrm{GeV}$ & $495.79 \mathrm{GeV}$ & $245.02 \mathrm{GeV}$ \\
\hline$T_{\rho}$ & $2.39 \times 10^{11} \mathrm{GeV}$ & $5.76 \times 10^{12} \mathrm{GeV}$ & $5.66 \times 10^{12} \mathrm{GeV}$ & $2.74 \times 10^{10} \mathrm{GeV}$ \\
\hline$T_{X}$ & $1.00 \times 10^{7} \mathrm{GeV}$ & $1.00 \times 10^{7} \mathrm{GeV}$ & $1.00 \times 10^{7} \mathrm{GeV}$ & $1.00 \times 10^{7} \mathrm{GeV}$ \\
\hline $\tan \beta$ & 3.69 & 3.93 & 3.43 & 4.04 \\
\hline$m_{h}$ & $126.20 \mathrm{GeV}$ & $125.89 \mathrm{GeV}$ & $124.65 \mathrm{GeV}$ & $124.03 \mathrm{GeV}$ \\
\hline$m_{\text {stop }}$ & $3.05 \mathrm{TeV}$ & $3.61 \mathrm{TeV}$ & $2.93 \mathrm{TeV}$ & $1.90 \mathrm{TeV}$ \\
\hline$X_{t}$ & $3.43 \times m_{\text {stop }}$ & $3.41 \times m_{\text {stop }}$ & $2.98 \times m_{\text {stop }}$ & $2.39 \times m_{\text {stop }}$ \\
\hline$|\mu|$ & $3.72 \mathrm{TeV}$ & $4.38 \mathrm{TeV}$ & $3.27 \mathrm{TeV}$ & $1.93 \mathrm{TeV}$ \\
\hline$|B|$ & $4.21 \mathrm{TeV}$ & $4.72 \mathrm{TeV}$ & $3.22 \mathrm{TeV}$ & $1.97 \mathrm{TeV}$ \\
\hline$\left|B_{\mathrm{EW}} / B\right|$ & 0.92 & 1.00 & 0.91 & 0.5 \\
\hline$\left|M_{3}\right|$ & $5.73 \times m_{3 / 2}$ & $5.73 \times m_{3 / 2}$ & $6.85 \times m_{3 / 2}$ & $7.83 \times m_{3 / 2}$ \\
\hline$\left|M_{2}\right|$ & $0.98 \times m_{3 / 2}$ & $0.98 \times m_{3 / 2}$ & $0.98 \times m_{3 / 2}$ & $0.98 \times m_{3 / 2}$ \\
\hline$\left|M_{1}\right|$ & $0.75 \times m_{3 / 2}$ & $0.75 \times m_{3 / 2}$ & $0.69 \times m_{3 / 2}$ & $0.64 \times m_{3 / 2}$ \\
\hline$m_{\widetilde{t}_{1}}$ & $3.52 \mathrm{TeV}$ & $4.12 \mathrm{TeV}$ & $3.31 \mathrm{TeV}$ & $2.15 \mathrm{TeV}$ \\
\hline$m_{\widetilde{t}_{2}}$ & $2.62 \mathrm{TeV}$ & $3.17 \mathrm{TeV}$ & $2.57 \mathrm{TeV}$ & $1.65 \mathrm{TeV}$ \\
\hline$m_{\widetilde{Q}_{L}}^{2}$ & $11.72 \mathrm{TeV}^{2}$ & $16.56 \mathrm{TeV}^{2}$ & $10.36 \mathrm{TeV}^{2}$ & $4.24 \mathrm{TeV}^{2}$ \\
\hline$m_{\widetilde{d}_{R}}^{2}$ & $15.97 \mathrm{TeV}^{2}$ & $22.52 \mathrm{TeV}^{2}$ & $13.60 \mathrm{TeV}^{2}$ & $5.40 \mathrm{TeV}^{2}$ \\
\hline$m_{l_{L}}^{2}$ & $0.78 \mathrm{TeV}^{2}$ & $0.93 \mathrm{TeV}^{2}$ & $0.44 \mathrm{TeV}^{2}$ & $0.18 \mathrm{TeV}^{2}$ \\
\hline$m_{\widetilde{e}_{R}}^{2}$ & $1.42 \mathrm{TeV}^{2}$ & $1.75 \mathrm{TeV}^{2}$ & $0.81 \mathrm{TeV}^{2}$ & $0.31 \mathrm{TeV}^{2}$ \\
\hline
\end{tabular}

Table 4. SUSY mass spectrums and parameters with $\Lambda_{\mathrm{GUT}}=2 \times 10^{16} \mathrm{GeV}$. Higgs mass is calculated by FeynHiggs [46, 58-61].

\section{B Concrete parameter set}

The parameter sets which predict $m_{h} \approx 125 \mathrm{GeV}$ are in table 4 . The Higgs mass is calculated by FeynHiggs $[46,58-61] . \quad m_{\widetilde{t}_{1,2}}$ are the stop masses in the mass eigenstate. $m_{\widetilde{Q}_{L}}^{2}, m_{\widetilde{d}_{R}}^{2}, m_{\widetilde{l}_{L}}^{2}$ and $m_{\widetilde{e}_{R}}^{2}$ are the soft SUSY breaking terms of the squarks $\left(\widetilde{Q}_{L}, \widetilde{d}_{R}\right)$ and sleptons $\left(\widetilde{l}_{L}, \widetilde{e}_{R}\right)$.

Open Access. This article is distributed under the terms of the Creative Commons Attribution License (CC-BY 4.0), which permits any use, distribution and reproduction in any medium, provided the original author(s) and source are credited. 


\section{References}

[1] CMS collaboration, Observation of a new boson at a mass of $125 \mathrm{GeV}$ with the CMS experiment at the LHC, Phys. Lett. B 716 (2012) 30 [arXiv:1207.7235] [INSPIRE].

[2] ATLAS collaboration, Observation of a new particle in the search for the standard model Higgs boson with the ATLAS detector at the LHC, Phys. Lett. B 716 (2012) 1 [arXiv: 1207.7214] [INSPIRE].

[3] Particle Data Group collaboration, K.A. Olive et al., Review of particle Physics, Chin. Phys. C 38 (2014) 090001 [InSPIRE].

[4] G.F. Giudice and A. Strumia, Probing high-scale and split supersymmetry with Higgs mass measurements, Nucl. Phys. B 858 (2012) 63 [arXiv:1108.6077] [InSPIRE].

[5] G. Degrassi et al., Higgs mass and vacuum stability in the standard model at NNLO, JHEP 08 (2012) 098 [arXiv:1205.6497] [INSPIRE].

[6] T. Hirayama, N. Ishimura and N. Maekawa, A model with simultaneous dynamical breaking of supersymmetry and GUT gauge symmetry, Prog. Theor. Phys. 101 (1999) 1343 [hep-ph/9805457] [INSPIRE].

[7] K. Agashe, GUT and SUSY breaking by the same field, Phys. Lett. B 444 (1998) 61 [hep-ph/9809421] [INSPIRE].

[8] K. Agashe, Improved GUT and SUSY breaking by the same field, Nucl. Phys. B 588 (2000) 39 [hep-ph/0003236] [INSPIRE].

[9] B. Bajc and G. Senjanović, Proton decay, supersymmetry breaking and its mediation, Phys. Lett. B 648 (2007) 365 [hep-ph/0611308] [INSPIRE].

[10] B. Bajc and A. Melfo, Metastable gauged O'Raifeartaigh, JHEP 04 (2008) 062 [arXiv:0801.4349] [INSPIRE].

[11] B. Bajc, S. Lavignac and T. Mede, Supersymmetry breaking induced by radiative corrections, JHEP 07 (2012) 185 [arXiv:1202.2845] [INSPIRE].

[12] N. Arkani-Hamed, J. March-Russell and H. Murayama, Building models of gauge mediated supersymmetry breaking without a messenger sector, Nucl. Phys. B 509 (1998) 3 [hep-ph/9701286] [INSPIRE].

[13] E. Poppitz and S.P. Trivedi, New models of gauge and gravity mediated supersymmetry breaking, Phys. Rev. D 55 (1997) 5508 [hep-ph/9609529] [INSPIRE].

[14] N. Haba, N. Maru and T. Matsuoka, Supersymmetry breaking without a messenger sector, Phys. Rev. D 56 (1997) 4207 [hep-ph/9703250] [INSPIRE].

[15] H. Murayama, A Model of direct gauge mediation, Phys. Rev. Lett. 79 (1997) 18 [hep-ph/9705271] [INSPIRE].

[16] M.A. Luty and J. Terning, New mechanisms of dynamical supersymmetry breaking and direct gauge mediation, Phys. Rev. D 57 (1998) 6799 [hep-ph/9709306] [InSPIRE].

[17] K. Agashe, An improved model of direct gauge mediation, Phys. Lett. B 435 (1998) 83 [hep-ph/9804450] [INSPIRE].

[18] R. Kitano, H. Ooguri and Y. Ookouchi, Direct mediation of meta-stable supersymmetry breaking, Phys. Rev. D 75 (2007) 045022 [hep-ph/0612139] [INSPIRE]. 
[19] H. Murayama and Y. Nomura, Gauge mediation simplified, Phys. Rev. Lett. 98 (2007) 151803 [hep-ph/0612186] [INSPIRE].

[20] C. Csáki, Y. Shirman and J. Terning, A simple model of low-scale direct gauge mediation, JHEP 05 (2007) 099 [hep-ph/0612241] [INSPIRE].

[21] N. Haba and N. Maru, A simple model of direct gauge mediation of metastable supersymmetry breaking, Phys. Rev. D 76 (2007) 115019 [arXiv:0709.2945] [INSPIRE].

[22] S.A. Abel, C. Durnford, J. Jaeckel and V.V. Khoze, Patterns of gauge mediation in metastable SUSY breaking, JHEP 02 (2008) 074 [arXiv:0712.1812] [INSPIRE].

[23] K.A. Intriligator, N. Seiberg and D. Shih, Dynamical SUSY breaking in meta-stable vacua, JHEP 04 (2006) 021 [hep-th/0602239] [INSPIRE].

[24] R. Dermisek, H.D. Kim and I.-W. Kim, Mediation of supersymmetry breaking in gauge messenger models, JHEP 10 (2006) 001 [hep-ph/0607169] [INSPIRE].

[25] L. Matos, Gauge Mediation with Gauge Messengers in SU(5), JHEP 12 (2010) 042 [arXiv: 1007.3616] [INSPIRE].

[26] H. Abe, T. Kobayashi and Y. Omura, Metastable supersymmetry breaking vacua from conformal dynamics, Phys. Rev. D 77 (2008) 065001 [arXiv: 0712.2519] [INSPIRE].

[27] H. Abe, T. Kobayashi and Y. Omura, R-symmetry, supersymmetry breaking and metastable vacua in global and local supersymmetric theories, JHEP 11 (2007) 044 [arXiv:0708.3148] [INSPIRE].

[28] H. Abe, T. Higaki, T. Kobayashi and Y. Omura, Moduli stabilization, F-term uplifting and soft supersymmetry breaking terms, Phys. Rev. D 75 (2007) 025019 [hep-th/0611024] [INSPIRE].

[29] G.F. Giudice and R. Rattazzi, Extracting supersymmetry breaking effects from wave function renormalization, Nucl. Phys. B 511 (1998) 25 [hep-ph/9706540] [INSPIRE].

[30] K. Intriligator and M. Sudano, General gauge mediation with gauge messengers, JHEP 06 (2010) 047 [arXiv: 1001.5443] [INSPIRE].

[31] J. Hisano, H. Murayama and T. Goto, Threshold correction on gaugino masses at grand unification scale, Phys. Rev. D 49 (1994) 1446 [InSPIRE].

[32] T. Goto and T. Nihei, Effect of RRRR dimension five operator on the proton decay in the minimal SU(5) SUGRA GUT model, Phys. Rev. D 59 (1999) 115009 [hep-ph/9808255] [INSPIRE].

[33] H. Murayama and A. Pierce, Not even decoupling can save minimal supersymmetric SU(5), Phys. Rev. D 65 (2002) 055009 [hep-ph/0108104] [INSPIRE].

[34] K.J. Bae, R. Dermisek, H.D. Kim and I.-W. Kim, Mixed Bino-Wino-Higgsino dark matter in gauge messenger models, JCAP 08 (2007) 014 [hep-ph/0702041] [INSPIRE].

[35] K. Choi, K.S. Jeong, T. Kobayashi and K.-i. Okumura, Little SUSY hierarchy in mixed modulus-anomaly mediation, Phys. Lett. B 633 (2006) 355 [hep-ph/0508029] [INSPIRE].

[36] H. Abe, T. Kobayashi and Y. Omura, Relaxed fine-tuning in models with non-universal gaugino masses, Phys. Rev. D 76 (2007) 015002 [hep-ph/0703044] [INSPIRE].

[37] F. Gabbiani, E. Gabrielli, A. Masiero and L. Silvestrini, A complete analysis of FCNC and $C P$ constraints in general SUSY extensions of the standard model,

Nucl. Phys. B 477 (1996) 321 [hep-ph/9604387] [INSPIRE]. 
[38] M.A. Luty and R. Sundrum, Supersymmetry breaking and composite extra dimensions, Phys. Rev. D 65 (2002) 066004 [hep-th/0105137] [INSPIRE].

[39] M. Luty and R. Sundrum, Anomaly mediated supersymmetry breaking in four-dimensions, naturally, Phys. Rev. D 67 (2003) 045007 [hep-th/0111231] [INSPIRE].

[40] R. Sundrum, 'Gaugomaly' mediated SUSY breaking and conformal sequestering, Phys. Rev. D 71 (2005) 085003 [hep-th/0406012] [inSPIRE].

[41] M. Ibe, K.-I. Izawa, Y. Nakayama, Y. Shinbara and T. Yanagida, Conformally sequestered SUSY breaking in vector-like gauge theories, Phys. Rev. D 73 (2006) 015004 [hep-ph/0506023] [INSPIRE].

[42] M. Ibe, K.-I. Izawa, Y. Nakayama, Y. Shinbara and T. Yanagida, More on conformally sequestered SUSY breaking, Phys. Rev. D 73 (2006) 035012 [hep-ph/0509229] [INSPIRE].

[43] M. Schmaltz and R. Sundrum, Conformal sequestering simplified, JHEP 11 (2006) 011 [hep-th/0608051] [INSPIRE].

[44] H. Murayama, Y. Nomura and D. Poland, More visible effects of the hidden sector, Phys. Rev. D 77 (2008) 015005 [arXiv: 0709.0775] [InSPIRE].

[45] M.S. Carena, M. Quirós and C.E.M. Wagner, Effective potential methods and the Higgs mass spectrum in the MSSM, Nucl. Phys. B 461 (1996) 407 [hep-ph/9508343] [INSPIRE].

[46] T. Hahn, S. Heinemeyer, W. Hollik, H. Rzehak and G. Weiglein, High-precision predictions for the light CP-even Higgs boson mass of the minimal supersymmetric standard model, Phys. Rev. Lett. 112 (2014) 141801 [arXiv:1312.4937] [INSPIRE].

[47] CMS collaboration, Precise determination of the mass of the Higgs boson and studies of the compatibility of its couplings with the standard model, CMS-PAS-HIG-14-009 (2014).

[48] T. Yanagida, Naturally light Higgs doublets in the supersymmetric grand unified theories with dynamical symmetry breaking, Phys. Lett. B 344 (1995) 211 [hep-ph/9409329] [INSPIRE].

[49] J. Hisano and T. Yanagida, An $N \neq 2$ SUSY gauge model for dynamical breaking of the grand unified SU(5) symmetry, Mod. Phys. Lett. A 10 (1995) 3097 [hep-ph/9510277] [INSPIRE].

[50] K.I. Izawa and T. Yanagida, $R$ invariant natural unification, Prog. Theor. Phys. 97 (1997) 913 [hep-ph/9703350] [INSPIRE].

[51] T. Watari and T. Yanagida, A solution to the doublet triplet splitting problem in the type IIB supergravity, hep-ph/0208107 [INSPIRE].

[52] M. Ibe and T. Watari, Upper bound of proton life time in product group unification, Phys. Rev. D 67 (2003) 114021 [hep-ph/0303123] [INSPIRE].

[53] T. Watari and T. Yanagida, Product group unification in type IIB string thoery, Phys. Rev. D 70 (2004) 036009 [hep-ph/0402160] [INSPIRE].

[54] F. Brümmer, M. Ibe and T.T. Yanagida, Focus point gauge mediation in product group unification, Phys. Lett. B 726 (2013) 364 [arXiv:1303.1622] [INSPIRE].

[55] H. Abe, T. Kobayashi, Y. Omura and T. Watanabe, work in progress.

[56] R. Blumenhagen, B. Körs, D. Lüst and S. Stieberger, Four-dimensional string compactifications with D-branes, orientifolds and fluxes, Phys. Rept. 445 (2007) 1 [hep-th/0610327] [INSPIRE]. 
[57] L.E. Ibanez and A.M. Uranga, String theory and particle physics: an introduction to string phenomenology, Cambridge University Press, Cambridge U.K. (2012).

[58] S. Heinemeyer, W. Hollik and G. Weiglein, FeynHiggs: a program for the calculation of the masses of the neutral CP even Higgs bosons in the MSSM,

Comput. Phys. Commun. 124 (2000) 76 [hep-ph/9812320] [INSPIRE].

[59] S. Heinemeyer, W. Hollik and G. Weiglein, The masses of the neutral CP-even Higgs bosons in the MSSM: accurate analysis at the two loop level, Eur. Phys. J. C 9 (1999) 343 [hep-ph/9812472] [INSPIRE].

[60] G. Degrassi, S. Heinemeyer, W. Hollik, P. Slavich and G. Weiglein, Towards high precision predictions for the MSSM Higgs sector, Eur. Phys. J. C 28 (2003) 133 [hep-ph/0212020] [INSPIRE].

[61] M. Frank et al., The Higgs boson masses and mixings of the complex MSSM in the Feynman-diagrammatic approach, JHEP 02 (2007) 047 [hep-ph/0611326] [INSPIRE]. 